\title{
A Determination of the Phase Diagram of Relaxed Langmuir Monolayers of Behenic Acid
}

\section{Citation}

Bommarito, G. M., W. J. Foster, Peter S. Pershan, and M. L. Schlossman. 1996. A determination of the phase diagram of relaxed Langmuir monolayers of Behenic acid. Journal of Chemical Physics 105(12): 5265-5285.

\section{Published Version}

doi:10.1063/1.472367

\section{Permanent link}

http://nrs.harvard.edu/urn-3:HUL.InstRepos:10357479

\section{Terms of Use}

This article was downloaded from Harvard University's DASH repository, and is made available under the terms and conditions applicable to Other Posted Material, as set forth at http:// nrs.harvard.edu/urn-3:HUL.InstRepos:dash.current.terms-of-use\#LAA

\section{Share Your Story}

The Harvard community has made this article openly available.

Please share how this access benefits you. Submit a story.

\section{Accessibility}




\title{
A determination of the phase diagram of relaxed Langmuir monolayers of Behenic acid
}

\author{
G. M. Bommarito, ${ }^{a), b)}$ W. J. Foster, ${ }^{\text {c) }}$ P. S. Pershan ${ }^{\text {a) }}$ \\ Department of Physics and Division of Applied Sciences, Harvard University, Cambridge, \\ Massachusetts 02138 \\ M. L. Schlossman \\ Department of Physics and Department of Chemistry, University of Illinois (M/C 273), Chicago, \\ Illinois 60607-7059
}

(Received 14 December 1995; accepted 6 May 1996)

\begin{abstract}
Grazing incidence x-ray scattering (GIXS) and Brewster angle microscopy (BAM) are used to determine the $\pi-T$ phase diagram of Behenic acid monolayers supported on the surface of water $(p \mathrm{H}=2.0)$ over the temperature range of $3{ }^{\circ} \mathrm{C}$ to $20.6{ }^{\circ} \mathrm{C}$. The phase diagram is constructed from measurements taken during isothermal compressions in which the surface pressure relaxed to a stable value at each surface density, and during temperature scans at fixed average surface density. The phase diagram is different than those previously reported for Behenic acid primarily because of the surface pressure relaxation. For temperatures less than $12{ }^{\circ} \mathrm{C}$ the phase diagram exhibits similar phases and topology as the published diagrams, although the location of the phases in the $\pi-T$ plane is different. Temperature scans combined with the isotherms, and the Clausius-Clapeyron relation are used to determine three coexistence lines that meet in a triple point. Changes in entropy across the phase boundaries are determined. Near room temperature $\left(20.6^{\circ} \mathrm{C}\right)$ only one phase is measured over the range of surface pressure from 0 dynes $/ \mathrm{cm}$ to the collapse pressure in contrast to reported measurements on monolayers out of equilibrium (i.e., when the surface pressure is not allowed to relax) that exhibit several phases near room temperature at pressures higher than the collapse pressure. Discrepancies are observed between the average area per molecule $\left(A_{T}\right)$ and the area per molecule determined from measurements of the unit cell $\left(A_{X}\right)$ in the close packed regions of the phase diagram. It is conjectured that the $\pi-A_{X}$ plane is a better representation of the ordered equilibrium monolayer phases than the $\pi-A_{T}$ plane. Isotherms plotted in the $\pi-A_{X}$ plane are used to determine the compressibility of the ordered phases and the nature of the phase transitions. (C) 1996 American Institute of Physics. [S0021-9606(96)00831-8]
\end{abstract}

\section{INTRODUCTION}

Langmuir monolayers of various materials have been the subject of many studies since Langmuir first demonstrated, in 1917, that amphiphilic organic molecules adsorbed at the air-water interface. ${ }^{1}$ The fact that researchers are still investigating these systems nearly a century later is indicative of a curious paradox. On one hand, Langmuir monolayers have tremendous appeal because they are widely thought of as simple models, useful in elucidating the structure-function relationship in much more complicated two-dimensional (2D) systems such as biological membranes or the surface of biological macromolecules. ${ }^{1-4}$ On the other hand, even the simplest Langmuir monolayers have presented many difficult fundamental questions, and are still far from being completely understood. In this regard, it has proven to be more difficult to obtain definitive results for Langmuir monolayers than for other systems exhibiting mesomorphic behavior, such as bulk thermotropic liquid crystals, for which the ex-

\footnotetext{
a) Authors to whom correspondence should be addressed.

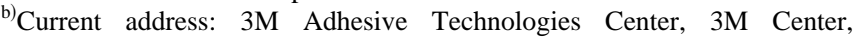
Building 236-GA-03, St. Paul, MN, 55144-1000; Electronic mail: gmbommarito@mmm.com

${ }^{c}$ Current address: Division of Experimental Medicine, Brigham and Women's Hospital LMRC301, 221 Longwood Avenue, Boston, MA 02115.
}

perimental facts and the theoretical framework are relatively well established. ${ }^{5,6}$ Among the various fundamental questions still in need of a clear and thorough answer is the issue of when Langmuir monolayers of long chain fatty acids are in thermodynamic equilibrium. The present study addresses this issue, and more specifically aims to determine fundamental features for one portion of the equilibrium phase diagram for a Langmuir monolayer of a long chain fatty acid.

Although many phase diagrams have been already proposed, ${ }^{7-13}$ it is not clear that any of these are truly equilibrium diagrams. The question arises because, in almost all of the phase diagrams published, the monolayer is compressed continuously, at a constant rate, without allowing sufficient time for the surface pressure $\pi$ to relax to an equilibrium value. That relaxation effects in Langmuir monolayers are important in establishing true equilibrium has already been demonstrated by several researchers. ${ }^{13-25}$ For example, Schlossman et al. showed that when a Langmuir monolayer of tetracosanoic acid $\left(\mathrm{CH}_{3}\left(\mathrm{CH}_{3}\right)_{22} \mathrm{COOH}\right)$ at $p \mathrm{H}=2.0$ and $25^{\circ} \mathrm{C}$, is allowed to relax completely following each incremental compression, both the isotherm and the x-ray scattering results are different from those that are obtained when the film is not allowed to equilibrate. ${ }^{21,22}$ Using infrared external reflection spectroscopy in comparison with x-ray dif- 
fraction, Buontempo et al. demonstrated that collective tilt ordering is different in relaxed and unrelaxed monolayers of heneicosanol $\left(\mathrm{CH}_{3}\left(\mathrm{CH}_{3}\right)_{20} \mathrm{OH}\right){ }^{23}$ Lin et al. found that with increasing time a Langmuir monolayer of heneicosanol relaxes from the uniaxially distorted pseudo hexagonal phase, that is initially formed upon constant compression to the desired surface pressure, to an undistorted hexagonal structure. ${ }^{24}$ Finally, Foster et al. determined the phase diagram for a relaxed Langmuir monolayer of methyl eicosanoate $\left(\mathrm{CH}_{3}\left(\mathrm{CH}_{2}\right)_{18} \mathrm{COOCH}_{3}\right)$; and found that although the topology of the relaxed phase diagram for this long chain fatty ester is in good agreement with previous work published for this system, the relaxed monolayer exhibits an additional phase previously unreported. ${ }^{25}$ An important difference between the work presented here and most of the others $^{7-13}$ is that thermodynamic information is collected simultaneously with $\mathrm{x}$-ray measurement of the microscopic structure, and both are combined to ensure that the results are self-consistent.

This paper describes an attempt to construct one section of an equilibrium phase diagram of a Langmuir monolayer using grazing incidence $\mathrm{x}$-ray scattering (GIXS). Specifically, Langmuir monolayers of Behenic acid $\left(\mathrm{CH}_{3}\left(\mathrm{CH}_{2}\right)_{20} \mathrm{COOH}\right)$ on an acidified water subphase $(p \mathrm{H}$ $=2.0, \mathrm{HCl}$ buffer) have been selected for study. The low $p \mathrm{H}$ of the subphase was chosen so as to keep the carboxyl head groups of the fatty acid protonated, thus maintaining neutrality of the monolayer. This enhances the stability of the monolayer phase, and is a common experimental condition when studying Langmuir monolayers of fatty acids. Qualitatively, the constructed phase diagram has a similar topology to that already reported in the literature. ${ }^{7-13}$ Quantitatively, there are differences due primarily to relaxation effects. The implications of our measurements with respect to the equilibrium nature of Langmuir monolayers will be discussed. In this respect, it is found that, even for relaxed films, anomalous effects characteristic of metastable monolayers are observed. This complicates interpretation of $\pi-A_{T}$ isotherms, where $A_{T}$ is the macroscopic area per molecule deposited on the trough. However, when the phase diagram is presented in terms of the microscopic area per molecule, $A_{X}$, extracted from the positions of the GIXS peaks, many of these difficulties are removed. In this way, it is possible to obtain a direct measurement of the slope and location of the various coexistence lines on the phase diagram. More importantly, the Clausius-Clapeyron equation is applied to calculate the entropy changes $(\Delta S)$ associated with observed phase transitions in which $\Delta A_{X}$ is directly measured. Although a similar approach has been employed by Berge et al. ${ }^{26}$ to calculate the entropy change upon melting of a monolayer of short chain alcohols at the equilibrium spreading pressure, $\pi_{\mathrm{ESP}}$ (i.e., at coexistence with the liquid bulk alcohol phase), we demonstrate here, for the first time, that this approach can also be used to study phase transitions of Langmuir monolayers, between two ordered (crystalline or hexatic) phases. The extracted values of $\Delta S$ are sensible when compared to those characteristic of analogous bulk and surface systems, allowing for a valuable elucidation of the equilibrium nature of the various phases observed.

This paper is divided into four main sections. Section II discusses the characteristics of the Langmuir trough used in these measurements, and outlines the various procedures employed to clean the trough and prepare fresh Langmuir monolayers prior to the X-ray measurements. Some background information describing the liquid surface spectrometer used in these experiments, and a discussion of GIXS are also included in this section. We also describe the differences between relaxation isotherms and constant compression isotherms, and conclude that the two compression methods produce markedly different isotherms. The most obvious of these differences being the much lower collapse pressure observed in the relaxation isotherms.

Section III covers the principal results of this work in three parts. The first part describes the $\pi-T$ phase diagram derived from the GIXS measurements, and shows that there is a clear quantitative difference between the phase diagram constructed in the present study and those already reported: the range of surface pressures over which we can observe the various phases is much smaller on our diagram. This is a direct result of the much lower collapse pressures exhibited by relaxed monolayers. The second part of this section describes various phase transitions as observed both upon relaxation isotherms and temperature scans. The principal result here is that the trough molecular area $A_{T}$ (as opposed to the x-ray molecular area $A_{X}$ ) is not an acceptable intensive thermodynamic variable of the system, making the $\pi-A_{T}$ couple a poor choice for representation of a thermodynamically significant Langmuir monolayer phase diagram. This conclusion leads directly to the final part of this section where it is proposed that the monolayer phase diagram can be represented in terms of $\pi$ and $A_{X}$. Isotherms plotted in the $\pi-A_{X}$ plane enable the determination of the compressibility of the ordered phases and the extent to which transitions are continuous or discontinuous in $A_{X}$. Temperature scans near a phase boundary combined with the Clausius-Clapeyron relation and X-ray measurements of $A_{X}$ can be used to calculate the entropy change accompanying the phase transitions. This thermodynamic analysis would not have been possible with the x-ray measurements represented on a $\pi-A_{T}$ phase diagram.

Section IV provides further details on monolayer thermodynamics and the determination of entropy changes across transitions between two ordered phases. In addition, this section lists some of the anomalous features observed in this study, providing an interpretation for the presence of these effects. We attempt to connect these anomalous effects to the observed discrepancies between $A_{T}$ and $A_{X}$.

Section $\mathrm{V}$ highlights the more interesting conclusions of this work.

\section{EXPERIMENTAL DETAILS}

\section{A. The Langmuir trough}

The Langmuir trough used in all of these experiments is described elsewhere. ${ }^{22,27,28}$ The reader is referred to the latest 
of these references ${ }^{28}$ for a detailed description, and the discussion here will describe only general properties of the trough. The trough was milled out from a single piece of Teflon and attached rigidly to a piece of gold coated OFHC copper, which acted as the bottom cooling plate. The Teflon barrier traveled along the trough's edges, confining the monolayer to a specified area. The trough resided in a sealed aluminum box (maintaining a nearly 100\% RH environment). Approximately $1 \mathrm{in}$. above the trough was located a second piece of gold coated OFHC copper, acting as the upper cooling plate. The box was designed so that one could add or subtract water, aspirate the surface, change the monolayer density, and measure the surface pressure and temperature without opening the box and disturbing the clean experimental environment of zero-grade nitrogen. The motor driving the barrier's motion was mounted outside the box enclosing the trough, and coupled to the barrier's translation stage using a Huntington rotational feedthrough designed for high vacuum systems. The lower and upper cooling plates as well as the aluminum box itself were temperature controlled to $0.25{ }^{\circ} \mathrm{C}$ using two Neslab closed cycle systems. The temperature of the subphase was measured directly using an Omega Teflon encased temperature probe immersed into the clean water behind the barrier. The box was covered with closed cell foam insulation, and temperature isolated from the kinematic mount of the liquid surface spectrometer using a Delrin spacer. Condensation was controlled by maintaining a temperature gradient between the lower and upper cooling plates of $2-3{ }^{\circ} \mathrm{C}$ (the top plate being warmer). The surface pressure was measured using the Wilhelmy plate method, ${ }^{16}$ with a resolution of 0.01 dynes $/ \mathrm{cm}$ and an accuracy of \pm 0.5 dynes $/ \mathrm{cm}$. The system as a whole was computer controlled using software already described elsewhere. ${ }^{27}$

\section{B. Materials and procedures}

Behenic acid obtained from Fluka was used without further purification. Baker Resi-analyzed chloroform was used as a solvent. Stringent cleaning procedures were observed in preparation for and during all experiments. Water for the experiments was obtained from a Millipore Milli-Q UV+ system fed by a Milli-RO system. The $p \mathrm{H}$ was adjusted to $2.0 \pm 0.1$ prior to use by adding Baker Ultrex II hydrochloric acid. The water was stored in a closed $4 \mathrm{~L}$ glass vessel and transferred to the trough using a closed system of PTFE tubing. Before each synchrotron run the apparatus described above was entirely disassembled, cleaned using detergent washing and solvent wiping, and reassembled in a clean hood. The Teflon trough and barrier were cleaned separately using a multistep procedure which included:

(i) Acetone and chloroform wiping.

(ii) Wash in a 50-50 mixture of sulfuric acid containing NO-CHROMIX and water (20-30 min).

(iii) Rinse in boiling Milli-Q water (20-30 min).

(iv) Continuous wash under running Milli-Q water at room temperature (30-40 $\mathrm{min})$.

The trough and barrier were mounted and sealed within the nitrogen purged aluminum box immediately following the procedure outlined above, thus minimizing the amount of time the trough was exposed to the ambient environment. Once in the box, the trough was repeatedly flushed with Milli-Q water by filling it and then removing the water using the built-in aspirating glass pipette. This was typically done 20-50 times a day over a period of 2 or 3 days before the system was clean enough to take isotherms. A clean water surface was prepared for deposition of a monolayer in the following manner. With the barrier at the largest surface area setting $\left(\approx 200 \mathrm{~cm}^{2}\right)$, the trough was filled with water to form a large meniscus above the trough's edges. The surface pressure was then allowed to equilibrate. At equilibrium, the barrier was moved to the end of the trough near the aspirator, at the smallest surface area setting $\left(\approx 50 \mathrm{~cm}^{2}\right)$. All the concentrated insoluble impurities were then vacuumed off the surface using the aspirator, while simultaneously setting the operational water level. At this point, the barrier was moved back to the largest surface area setting, and the surface pressure was allowed to re-equilibrate. Prior to spreading a monolayer, the cleanliness of the freshly prepared water surface was checked by reducing the surface area by a factor of 4. If the surface tension change produced by this compression was less than 0.08 dynes $/ \mathrm{cm}$, and remained stable for a period of $30 \mathrm{~min}$, the surface was considered to be clean enough to use. Deposition of a new monolayer was carried out using a Hamilton microliter syringe with a Teflon tipped piston. The stock solution of solvent and fatty acid was added to the water surface by carefully touching the drop hanging from the end of the needle to the water surface without submerging the needle tip. The newly deposited film was then allowed to relax and come to equilibrium by monitoring the surface pressure as a function of time. This initial equilibration typically occurred within 15 to $30 \mathrm{~min}$. The deposition procedure outlined above was found to result in area per molecule variations of less than approximately 5\%. Whenever flushing, aspirating, or depositing a new monolayer, the box was continuously purged using zero-grade nitrogen. At all other times, the enclosure was overpressurized to approximately 1 psi with zero-grade nitrogen.

\section{X-ray background}

\section{Liquid surface spectrometer}

The X-ray measurements were made at beam line X22B of the National Synchrotron Light Source at Brookhaven National Laboratory. The data reported here are the result of more than six months of beam time over a period of two years and represent detailed studies of at least 30 different monolayers.

The liquid surface spectrometer used for these studies has been described in detail elsewhere, ${ }^{29,30}$ and the reader is referred to these references for a complete description. A few details of interest are given here. The focused beam at the sample had an angular divergence of $1 \times 10^{-4}$ radians in the vertical and $1 \times 10^{-3}$ radians in the horizontal. The distances from the second monochromator crystal [a single bounce Ge(111) crystal used to reflect the beam down to the desired 


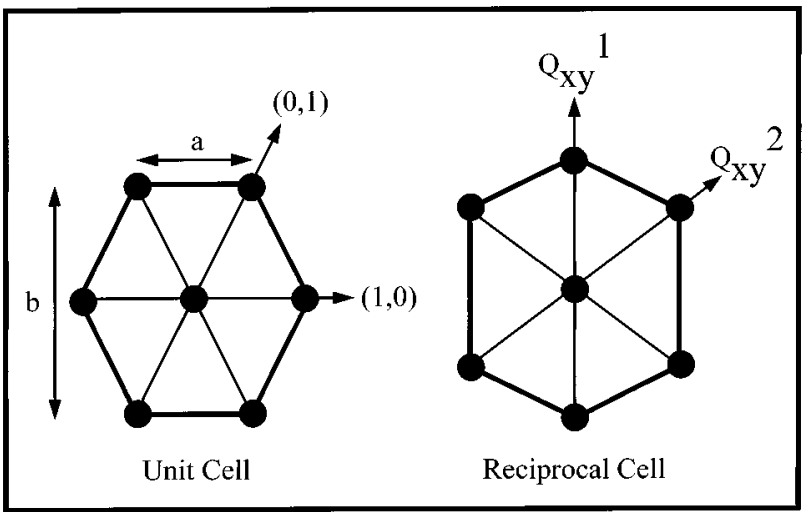

FIG. 1. Illustration of the convention to be used in this paper for describing unit cell dimensions for Langmuir monolayer phases. For an undistorted triangular lattice the ratio $b=\sqrt{3} a$. If the alkane chains are tilted towards the nearest-neighbor, say along the $(1,0)$ direction, then the maximum intensity for the peak at $Q_{x y}^{1}$ would be in the plane of the figure; the maximum intensity for the peak at $Q_{x y}^{2}$, as well as for the equivalent degenerate peak, would be out of plane of the figure. If the alkane chains are tilted between nearest neighbors, as along the $(-1,1)$ direction, the maximum intensity for both $Q_{x y}^{1}$ and $Q_{x y}^{2}$ would be out of the plane; however, the $Q_{z}$ value of the $Q_{x y}^{1}$ peak would be twice that of the $Q_{z}$ value of the $Q_{x y}^{2}$ peak.

angle of incidence with respect to the water surface] to sample and from sample to detector were both $600 \mathrm{~mm}$. In all diffraction measurements, Soller slits, with the leaves oriented vertically, were placed just before the detector. The horizontal angular spread accepted by the Soller slits was $3 \times 10^{-3}$ radians implying an in-plane resolution of $\Delta Q z$ $\approx 0.024 \AA^{-1}$. A Braun quartz wire position sensitive detector was placed behind the Soller slits with the wire oriented vertically, enabling measurements to be simultaneously recorded over a range of reflection angles with respect to the water surface, with a maximum possible angular resolution from a point scatterer of $8 \times 10^{-5}$ radians. For the present measurements this fine resolution was not necessary, and the 1024 channels electronically available for the $50 \mathrm{~mm}$ length of wire were combined into 4 bins of 256 channels each. This binning resulted in an angular resolution of 0.02 radians or $\Delta Q_{z}=0.06 \AA^{-1}$. With a wavelength of $1.527 \pm 0.06 \AA$, and an incident angle of $0.13^{\circ}$ (slightly less than the critical angle for total external reflection from water $0.15^{\circ}$ ), the spectrometer can access approximately $-2 \leqslant Q_{x y} \leqslant 2 \quad \AA^{-1}$ and $0 \leqslant Q_{z} \leqslant 1 \AA^{-1}$.

\section{Grazing incidence $x$-ray diffraction}

The techniques by which GIXS is used to determine unit cell dimensions and molecular orientation in Langmuir monolayers is now well established. ${ }^{29,31,32}$ The notation used throughout this paper to describe the dimensions of the 2Dunit cell is illustrated in Fig. 1. The samples are essentially two-dimensional powders and so the GIXS structure of the Langmuir monolayer phases can be characterized by the inplane, or $Q_{x y}$, and out of plane, $Q_{z}$, coordinates of the observed peaks. In this study, as with most others involving fatty acids, the unit cells for the primary phases possess a

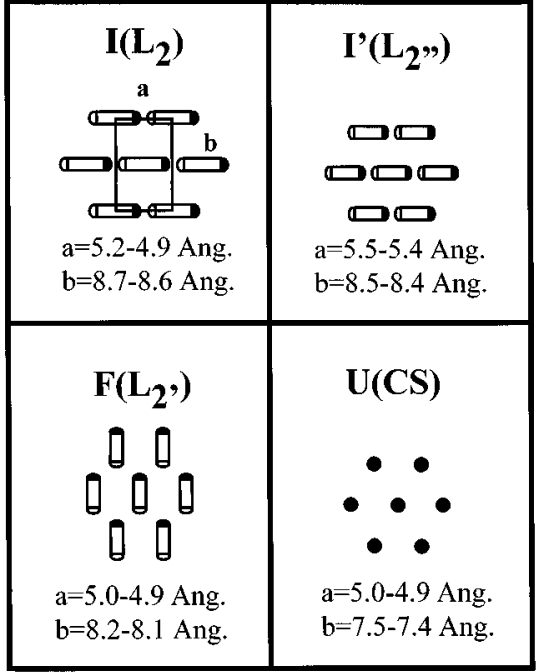

FIG. 2. Schematic representation of the in-plane packing for the various phases observed in this work. The associated lattice parameters $(a, b)$ are also given. The range of values specified for each parameter represent the changes observed in going from an uncompressed to a compressed lattice.

mirror plane and can be characterized by two first order peaks with coordinates $\left(Q_{x y}^{i}, Q_{z}^{i}\right)$ with $i=1,2$. In the present study, four distinct phases have been identified, although two of them appear to have the same point group.

These phases have been labeled by adopting a nomenclature analogous to that used to describe smectic liquid crystal phases with the same in-plane structure, ${ }^{21,22,33}$ however, to avoid confusion, the Stenhagen notation ${ }^{7}$ has been included in parenthesis. As shown in Fig. 2, the alkane chains of the $I\left(L_{2}\right)$ and $I^{\prime}\left(L_{2^{\prime \prime}}\right)$ phases are tilted towards a nearest neighbor, while for the $F\left(L_{2^{\prime}}\right)$ phase the tilt is in a direction between a nearest neighbor; the $U(C S)$ phase is untilted. This study did not distinguish between the two possible untilted phases, $U(C S)$ and $U^{\prime}(S)$, and all regions in which untilted phases were observed will be labeled as $U(C S){ }^{10,32}$ Table I lists the alternative nomenclature used to identify the observed phases elsewhere in the literature and summarizes the parameters characterizing each phase. In addition, Fig. 2 schematically depicts the in-plane packing of these phases.

\section{Relaxation isotherms}

Except for the few cases in which data were taken as a function of temperature, at fixed trough area, all of the GIXS data were taken during relaxation isotherms. Relaxation isotherms consist of a sequence of small trough area compressions separated by variable waiting periods. ${ }^{21,22}$ During the waiting period, the surface pressure was recorded at intervals of 1 to 2 min and the next compression occurred only if five successive measurements of $\pi$ were equal to one another within 0.01 dynes $/ \mathrm{cm}$. This criterion allowed enough time for relaxation processes with decay times on the order of a few hours, to come to a steady-state value of $\pi$. This was evident when comparing relaxation isotherms measured us- 
TABLE I. In-plane location $\left(Q_{x y}\right)$ of the diffraction peaks characterizing the various monolayer phases observed for fatty acids, in this and other pertinent works. The GIXS peaks with maximum intensity at $Q_{z}>0$ are in italic, while the in-plane peaks $\left(Q_{z}=0\right)$ are in normal type. Doubly degenerate peaks are indicated by (2) following the quoted value of $Q_{x y}$. All values have units of $\AA^{-1}$. A range of values indicates the change in $Q_{x y}$ observed upon compression of the film.

\begin{tabular}{|c|c|c|c|c|c|}
\hline \multirow{2}{*}{$\frac{\text { Nomenclature }}{\text { This work }}$} & \multicolumn{5}{|c|}{ Monolayer phase } \\
\hline & $I$ & $I^{\prime}$ & $F$ & $U^{\prime}$ & $U$ \\
\hline Ref. 9 & $D$ & $B$ & $C$ & $A^{\prime}$ & $A$ \\
\hline Ref. 7 & $L_{2}$ & $L_{2^{\prime \prime}}$ & $L_{2}$ & $C S$ & $S$ \\
\hline \multicolumn{6}{|c|}{ Chain Length } \\
\hline 20 & $1.48-1.51$ & $\ldots$ & & & \\
\hline Ref. 51 & $1.46-1.50(2)$ & $\cdots$ & $\cdots$ & $\cdots$ & $\cdots$ \\
\hline 21 & 1.44 & 1.68 & $1.505(2)$ & 1.67 & 1.68 \\
\hline Ref. 9 & $?$ & $1.51(2)$ & 1.66 & $1.51(2)$ & $1.51(2)$ \\
\hline 22 & 1.42 & & $1.48(2)$ & 1.62 & 1.68 \\
\hline Ref. 10 & $?$ & $\cdots$ & 1.57 & $1.50(2)$ & $1.51(2)$ \\
\hline 22 & $1.42-1.44$ & $1.68-1.695$ & $1.46-1.48(2)$ & & $1.64-1.66$ \\
\hline This work & $1.40-1.46(2)$ & $1.40-1.45(2)$ & $1.53-1.57$ & $\cdots$ & $1.51(2)$ \\
\hline 24 & $1.44-1.46$ & $\ldots$ & $1.49-1.50(2)$ & 1.66 & $\ldots$ \\
\hline Ref. 22 & $1.42-1.47(2)$ & & $1.54-1.60$ & $1.52(2)$ & \\
\hline
\end{tabular}

ing the above criterion to relaxation isotherms measured by allowing each relaxation to continue undisturbed for several hours. The final steady state values of $\pi$ were equivalent for both of these measurement modes. The relaxation phase diagram for behenic acid was constructed from the $\pi-T$ coordinates corresponding to phases identified from GIXS measurements after the surface pressure had reached a steadystate value. Complete measurements for a single temperature taken in this manner could occupy as long as one or two days. As a result, stability and cleanliness of the system over this time frame was of the utmost importance. The requirement for stability precluded use of a material that shows appreciable solubility in the subphase. In this sense, Behenic acid was found to be very stable when spread on a highly

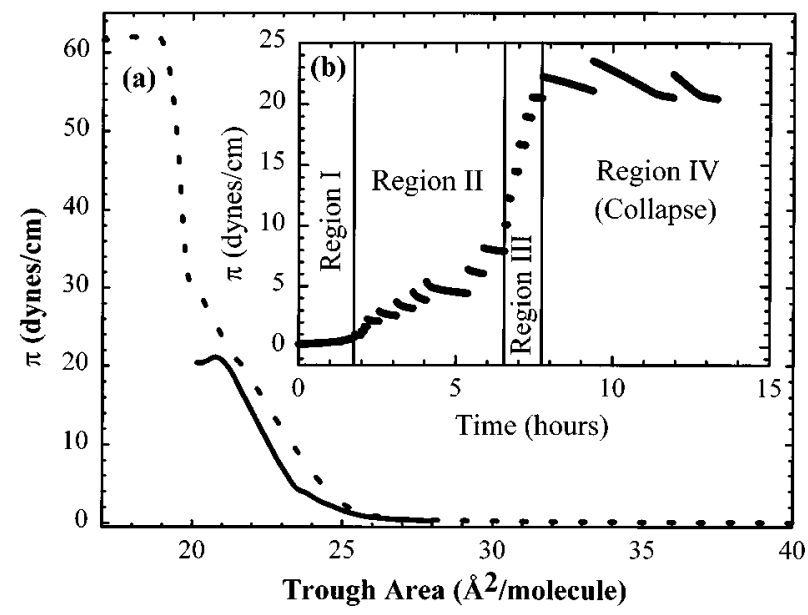

FIG. 3. (a) Comparison of a constant rate of compression isotherm (dashed line) and a relaxation isotherm (solid line) for a monolayer of Behenic acid at $20^{\circ} \mathrm{C}$ on a $0.1 \mathrm{M} \mathrm{HCl}$ aqueous subphase. The rate of compression for the constant compression isotherm was $8 \AA^{2} /$ min. (b) This inset shows the time trace of the relaxation isotherm. Each segment on the time trace corresponds to the relaxation in $\pi$ after a compression step. The four regions (I-IV) identified are discussed in the text. acidic aqueous subphase (i.e., $p \mathrm{H}=2.0$ or less). Figure 3(b) shows the surface pressure versus time trace for a relaxation isotherm at $20^{\circ} \mathrm{C}$. Referring to this figure, three distinct relaxation regimes, prior to collapse, were observed. At low surface pressures [below 1-2 dynes/cm, region I on Fig. $3(\mathrm{~b})]$, the relaxations are very quick (a few seconds), and are not readily visible with the displayed resolution. In the pressure range from 2 to 8 dynes/cm (region II), relaxations become very slow with decay times of hours. Most of the time, the decay in this region is well-described by an exponential form. Overall changes in the surface pressure within each relaxation in this intermediate pressure regime can be as large as 1-2 dynes/cm. At higher pressures (region III), the relaxations are completed after only a few minutes. To confirm that the monolayer was fully relaxed in this region of the isotherm, we measured several isotherms where each relaxation in region III was followed for several hours. The steady-state values of $\pi$ in both cases were identical. However, in region III, the exponential form is, in general, no longer a good description of the decay process. This kinetic behavior, exhibiting fast to slow to fast relaxations (regions I-III) as a function of increasing surface pressure, was observed in all films of Behenic acid explored in this work. Finally, in region IV the monolayer collapses and relaxation times increase drastically.

This relaxation isotherm should be compared to one that is typical of the more common isotherms reported in the literature, in which the film is continuously compressed while instantaneously measuring $\pi .^{21,22}$ For $T=20^{\circ} \mathrm{C}$, comparison between isotherms taken by these two different methods, as shown in Fig. 3(a), demonstrates that constant compression isotherms differ markedly from relaxation isotherms. For example, the collapse pressure for a constant compression isotherm can be as high as 60 dynes/cm! In relaxation isotherms, the maximum surface pressures reachable prior to collapse vary over the range of 11 to 20 dynes/ $\mathrm{cm}$, depending upon the temperature. Generally, constant 
compression isotherms show higher collapse pressures for a given trough area and temperature. Finally, in constant compression, isotherm features taken to indicate phase transitions (i.e., kinks or plateaus) are observed for pressures larger than the collapse pressure of a relaxed monolayer. Analogous features are missing in the relaxation isotherms. However, relaxation isotherms can exhibit other anomalous features [such as the discontinuous change in compressibility at 23.5 $\AA^{2} / \mathrm{mol}$ and 4 dynes/cm in Fig. 3(a)], that seem to be washed out in the constant compression isotherm counterpart. These effects will be discussed later.

\section{EXPERIMENTAL RESULTS}

\section{A. The $\pi-T$ phase diagram}

Figure 4 shows one of the principal results of the present study, namely the surface pressure $(\pi)$ vs temperature phase diagram for Behenic acid on water $(p \mathrm{H}=2.0, \mathrm{HCl}$ buffer $)$, constructed from GIXS measurements. The observations and analysis upon which this diagram is based will be described below. As was mentioned above, the general morphology is similar to phase diagrams that have been previously presented by others. However, there are significant quantitative differences that will be discussed. Regions occupied by the four distinct phases, $I\left(L_{2}\right), I^{\prime}\left(L_{2^{\prime \prime}}\right), F\left(L_{2^{\prime}}\right)$, and $U(C S)$, are indicated on the diagram.

The $I\left(L_{2}\right)$ phase occupies the lower right hand corner of the phase diagram above temperatures of $4.9 \pm 0.25^{\circ} \mathrm{C}$, and extends to pressures as high as 11.5 dynes $/ \mathrm{cm}$ at $12{ }^{\circ} \mathrm{C}$. Structurally, this phase is characterized by a collective molecular tilt toward the nearest-neighbor (designated as $\mathrm{NN}$ ). For an uncompressed film, the average value of the molecular tilt, $\theta$, is $32.5^{\circ}$; however, $\theta$ as small as $\sim 26.5^{\circ}$ has been observed when the $I\left(L_{2}\right)$ phase is compressed. The molecular area calculated from the observed diffraction peaks (heretofore referred to as x-ray area or $A_{X}$ ), ranges from $22.9 \pm 0.2$ $\AA^{2} / \mathrm{mol}$ for an uncompressed film, to $21.4 \pm 0.2 \AA^{2} / \mathrm{mol}$ at the highest surface pressure attainable while in this phase. The rectangular unit cell axis ratio $b / a$ [see Fig. 5(a) and (b)] for the uncompressed lattice is on average $1.68 \pm 0.02$, corresponding to distorted hexagonal, with the distortion extending along the tilt direction $(1,0)$. As the $I\left(L_{2}\right)$ phase is compressed, this ratio passes through a value of $\sqrt{3}=1.732$, indicating perfect hexagonal packing, even though the tilt breaks the hexagonal symmetry of the monolayer. Further compression again produces a distorted lattice with a maximum $b / a$ ratio in excess of $\sqrt{3}=1.732$, indicating that the cell is now extended perpendicular to the tilt direction. For high enough temperatures, where compression induces a transition from the $I\left(L_{2}\right)$ to the $F\left(L_{2}{ }^{\prime}\right)$ phase the ratio of $b / a$ grows to a maximum of $1.77 \pm 0.02$ within the $I\left(L_{2}\right)$ phase. It is also important to point out that at room temperature $\left(20.6{ }^{\circ} \mathrm{C}\right)$ the $I\left(L_{2}\right)$ phase is the only phase observed. Although previously published work ${ }^{10}$ has described the existence of other phases at higher pressures in this temperature region, they are not seen in relaxed monolayers for which the $I\left(L_{2}\right)$ phase is the only stable phase from a pressure of 0 dynes/cm up to the collapse pressure of approximately 9
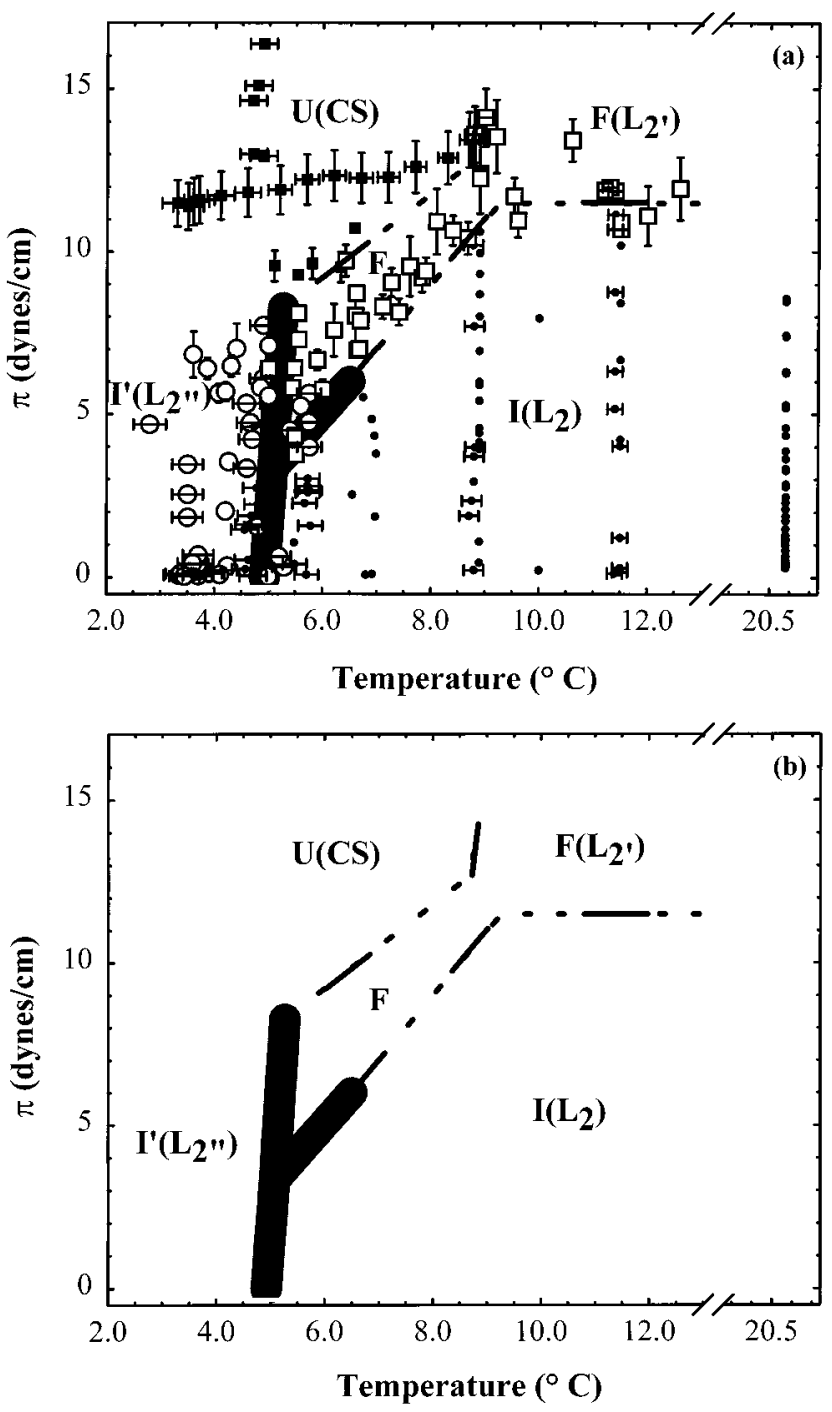

FIG. 4. (a). Surface pressure $\pi$ vs temperature phase diagram for Behenic acid on a $0.1 \mathrm{M} \mathrm{HCl}$ aqueous subphase. An x-ray measurement was made at each symbol. The various phases identified within this phase space are represented using different symbols. The $I\left(L_{2}\right)$ phase is shown as filled circles, the $I^{\prime}\left(L_{2^{\prime \prime}}\right)$ phase appears as open circles, the open squares represent the $F\left(L_{2^{\prime}}\right)$ phase, and the $U(C S)$ phase is depicted using filled squares. The thick shaded lines locate the $I^{\prime}\left(L_{2^{\prime \prime}}\right) / I\left(L_{2}\right), I^{\prime}\left(L_{2^{\prime \prime}}\right) / F\left(L_{2^{\prime}}\right)$, and $I\left(L_{2}\right) / F\left(L_{2^{\prime}}\right)$ boundaries about the $I^{\prime}\left(L_{2^{\prime \prime}}\right) / I\left(L_{2}\right) / F\left(L_{2^{\prime}}\right)$ triple point. Their thickness is representative of the uncertainty associated with the position of these boundaries in the vicinity of the triple point. The dashed lines show the position of the $I\left(L_{2}\right) / F\left(L_{2^{\prime}}\right)$ and $U(C S) / F\left(L_{2^{\prime}}\right)$ phase boundaries. These boundaries are shown as straight dashed lines because precise measurements of their curvature were not made. Solid segments along these dashed lines identify where an isotherm measurement was observed and followed across the boundary. Note that the temperature axis is broken at $13{ }^{\circ} \mathrm{C}$, so as to show the room temperature $\left(20.6{ }^{\circ} \mathrm{C}\right)$ data. (b). For added clarity, this figure reproduces only the phase boundaries observed in the surface pressure $\pi$ vs temperature phase diagram presented in (a).

dynes/cm (see Fig. 4). The collapse pressures measured vary between 9 and 15 dynes/cm over the temperature range of $3{ }^{\circ} \mathrm{C}$ to $20.6{ }^{\circ} \mathrm{C}$. It is likely that similar collapse pressures will occur over the range of $25^{\circ} \mathrm{C}$ to $30^{\circ} \mathrm{C}$. Because of this, we suspect that the rotator phases previously reported ${ }^{10}$ at these higher temperatures, at pressures above the collapse 

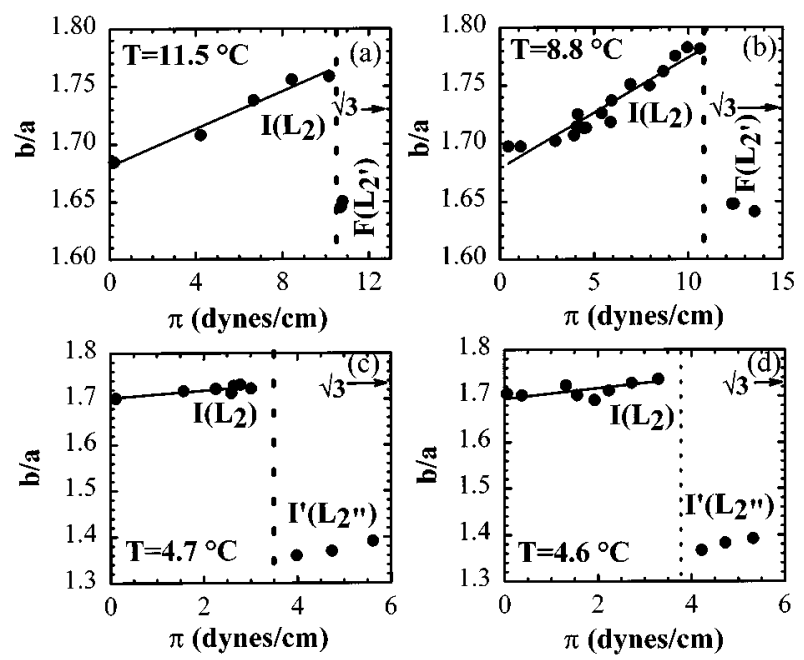

FIG. 5. Changes in the $b / a$ ratio upon compression from the $I\left(L_{2}\right)$ to the $F\left(L_{2^{\prime}}\right)$ phase at $11.5^{\circ} \mathrm{C}$ and $8.8^{\circ} \mathrm{C}\left[(\mathrm{a})\right.$ and (b)], and from the $I\left(L_{2}\right)$ to the $I^{\prime}\left(L_{2^{\prime \prime}}\right)$ phase at $4.7^{\circ} \mathrm{C}$ and $4.6^{\circ} \mathrm{C}[(\mathrm{c})$ and $(\mathrm{d})]$. Note that the transitions are sharp and without evidence of coexistence. A value of $b / a$ equal to $\sqrt{3}$ indicates perfect hexagonal packing.

pressure would not be seen in relaxed monolayers of behenic acid.

The $I^{\prime}\left(L_{2^{\prime \prime}}\right)$ phase occupies the lower left hand corner of the phase diagram, for $T \leqslant 5^{\circ} \mathrm{C}$ and $\pi \leqslant 8-9$ dynes $/ \mathrm{cm}$. The phase is similar to the $I\left(L_{2}\right)$ phase in that it is also tilted toward the $(1,0)$ nearest neighbor; however, the distortion of the $2 \mathrm{D}$ triangular packing in the tilt direction is much larger than for the $I\left(L_{2}\right)$ phase. Typical values of the $b / a$ ratio vary from $1.35 \pm 0.02$ at low $\pi$ to $1.40 \pm 0.02$ after compression [see Fig. 5(c) and (d)]. In the uncompressed state $\theta$ is $26.5^{\circ}$; however, values as small as $20^{\circ}$ are observed after compression. Similarly, in the uncompressed state $A_{X}$ is $20.9 \pm 0.2$ $\AA^{2} / \mathrm{mol}$; however, values as small as $19.4 \pm 0.2 \AA^{2} / \mathrm{mol}$ are observed after compression of the $I^{\prime}\left(L_{2^{\prime \prime}}\right)$ phase.

The $F\left(L_{2^{\prime}}\right)$ phase is tilted toward the next nearest neighbor [designated as NNN and perpendicular to the $(0,1)$ direction indicated in Fig. 1]. The hexagonal unit cell is distorted in the direction of the tilt with a $b / a$ ratio of $1.65 \pm 0.02$ [see Fig. 5(a) and (b)], and, over the range of $F\left(L_{2^{\prime}}\right)$ existence, it does not vary with $\pi$ and temperature beyond the established error bars. The molecular area can vary from $20.9 \pm 0.2$ $\AA^{2} /$ mol at the lowest pressures for which this phase can be observed, to $20.0 \pm 0.2 \AA^{2} / \mathrm{mol}$ as it is compressed. Concurrently, $\theta$ changes from $22.5^{\circ}$ to $19^{\circ}$.

At higher pressures, and temperatures lower than $\sim 9{ }^{\circ} \mathrm{C}$, the $F\left(L_{2^{\prime}}\right)$ phase transforms to the untilted $U(C S)$ phase. However, at temperatures above $11^{\circ} \mathrm{C}$ and the highest pressures, the monolayers are often not stable, and this instability is presumed to be a collapse into the third dimension. Although the $F\left(L_{2^{\prime}}\right)$ phase is regularly observed, the boundary between regions of instability and thermodynamic equilibrium is not precisely located.

The $I^{\prime}\left(L_{2^{\prime \prime}}\right)$ and $F\left(L_{2^{\prime}}\right)$ phases are separated by a steep boundary joining the $I\left(L_{2}\right) / I^{\prime}\left(L_{2^{\prime \prime}}\right) / F\left(L_{2^{\prime}}\right)$ triple point at $3.5 \pm 0.5 \mathrm{dynes} / \mathrm{cm}$ and $5.0 \pm 0.5^{\circ} \mathrm{C}$, and what is probably an upper triple point around $8.3 \pm 1.0$ dynes $/ \mathrm{cm} . .^{10,9,32,34}$ Temperature scans at fixed $A_{T}$ were used to follow the essentially vertical $I^{\prime}\left(L_{2^{\prime \prime}}\right) / F\left(L_{2^{\prime}}\right)$ phase boundary at approximately $5{ }^{\circ} \mathrm{C}$ from $\pi=4$ to 8.5 dynes $/ \mathrm{cm}$, (these values being uncertain to approximately \pm 0.5 dyne $/ \mathrm{cm}$ ); while the $I\left(L_{2}\right) / F\left(L_{2^{\prime}}\right)$ boundary was located through a series of relaxation isotherms at various temperatures between $5.5^{\circ} \mathrm{C}$ and $15{ }^{\circ} \mathrm{C}$. However, supercooling effects (to be discussed shortly) made it difficult to accurately identify the location of $I\left(L_{2}\right) / I^{\prime}\left(L_{2^{\prime \prime}}\right)$ phase boundary directly from GIXS observations, during scans of temperature at fixed $A_{T}$. Furthermore, small temperature drifts made it difficult to obtain precise measurements near the $I\left(L_{2}\right) / I^{\prime}\left(L_{2^{\prime \prime}}\right) / F\left(L_{2^{\prime}}\right)$ triple point. It will be explained in the discussion and interpretation how, by application of the Clausius-Clapeyron relation and the measured location and slopes of the $I^{\prime}\left(L_{2^{\prime \prime}}\right) / F\left(L_{2^{\prime}}\right)$ and $I\left(L_{2}\right) / F\left(L_{2^{\prime}}\right)$ phase boundaries away from the triple point, the $I\left(L_{2}\right) / I^{\prime}\left(L_{2^{\prime \prime}}\right) / F\left(L_{2^{\prime}}\right)$ triple point and the $I\left(L_{2}\right) / I^{\prime}\left(L_{2^{\prime \prime}}\right)$ boundary were located.

Returning to the discussion of supercooling effects observed at the $I\left(L_{2}\right) / I^{\prime}\left(L_{2^{\prime \prime}}\right)$ boundary, we specifically observed that in temperature scans at $\pi \approx 0$ dynes $/ \mathrm{cm}$, it was often possible to supercool a monolayer spread in the $I\left(L_{2}\right)$ phase to temperatures $1.3{ }^{\circ} \mathrm{C}$ lower than the $I\left(L_{2}\right) / I^{\prime}\left(L_{2^{\prime \prime}}\right)$ boundary before observing the transition in GIXS to the $I^{\prime}\left(L_{2^{\prime \prime}}\right)$ phase. It was also possible to spread a metastable film into the $I\left(L_{2}\right)$ phase at temperatures below that point identified in Fig. 4 as the $I^{\prime}\left(L_{2^{\prime \prime}}\right) / I\left(L_{2}\right)$ boundary.

On the other hand, Brewster angle microscopy (BAM) observations upon heating films (to be described below) spread at low temperatures and finite values of $\pi$ do show the $I^{\prime}\left(L_{2^{\prime \prime}}\right) / I\left(L_{2}\right)$ transition at the position indicated on the phase diagram. Referring to Fig. 6 , the transition can be seen at $\pi \approx 2.7$ dynes/cm by a drastic change in the coarseness of the powder as a $1.4{ }^{\circ} \mathrm{C}$ step between 4.7 and $6.1{ }^{\circ} \mathrm{C}$ is made. Furthermore, image analysis of the various domains visible in such images shows a quantitative $\mathrm{e}^{35}$ increase in the birefringence in going from 4.7 to $6.1{ }^{\circ} \mathrm{C}$, consistent with the increase in the average $\theta$ of the monolayer that is seen in GIXS measurements of the transition between the $I^{\prime}\left(L_{2^{\prime \prime}}\right)$ and $I\left(L_{2}\right)$ phases. Supercooling effects were not observed at the $I^{\prime}\left(L_{2^{\prime \prime}}\right) / F\left(L_{2^{\prime}}\right)$ boundary.

Completing the description of the $\pi-T$ phase diagram, the $U(C S)$ phase is observed to occupy the upper left hand corner of our diagram. In this study, the $I^{\prime}\left(L_{2^{\prime \prime}}\right)$ to $U(C S)$ transition was only measured with a limited number of isotherms and the position of the phase boundary has not been determined precisely. In most published reports using constant compression rate isotherms, the $I^{\prime}\left(L_{2^{\prime \prime}}\right) / U(C S)$ coexistence curve is shown to be nearly horizontal or with a small positive slope. ${ }^{10,11}$ However, some researchers have also depicted this boundary with a negative slope. ${ }^{13}$ The literature ${ }^{8,10,36}$ also suggests that at slightly higher temperature there should be another nearly vertical phase boundary separating slightly different untilted $U(C S)$ and $U^{\prime}(S)$ phases. This boundary is usually presented as extending up- 


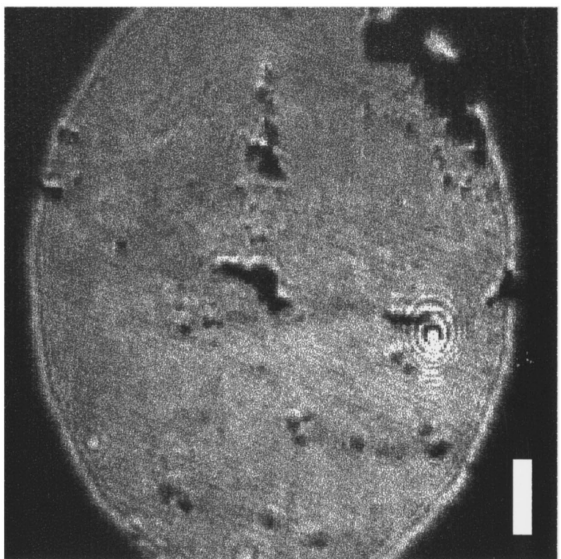

(A)

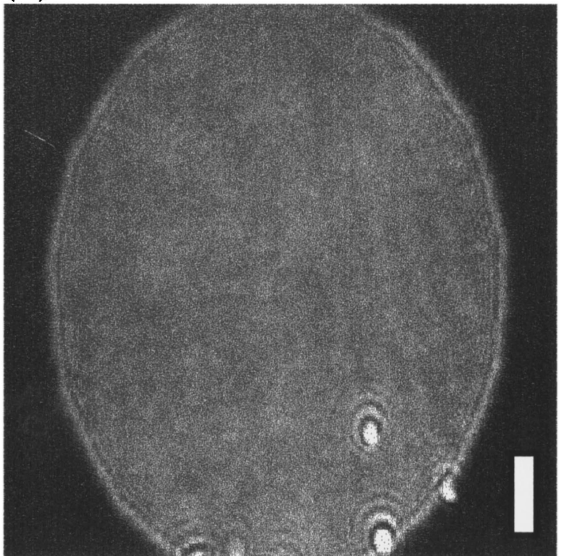

(C)

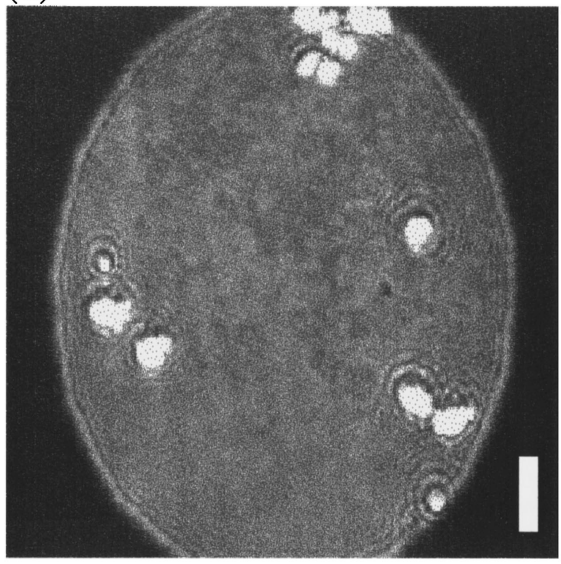

(E)

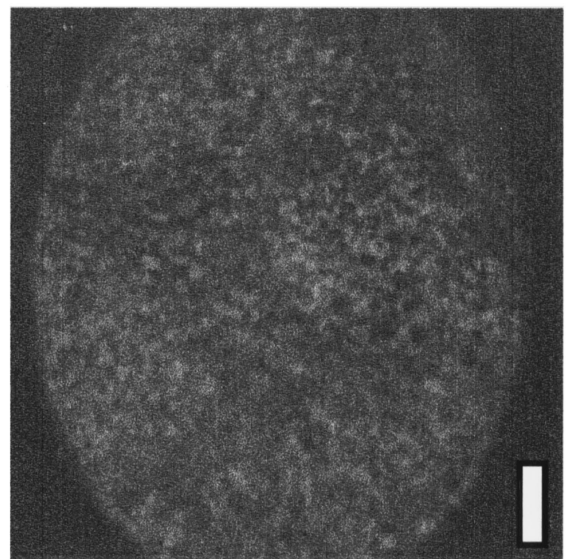

(B)

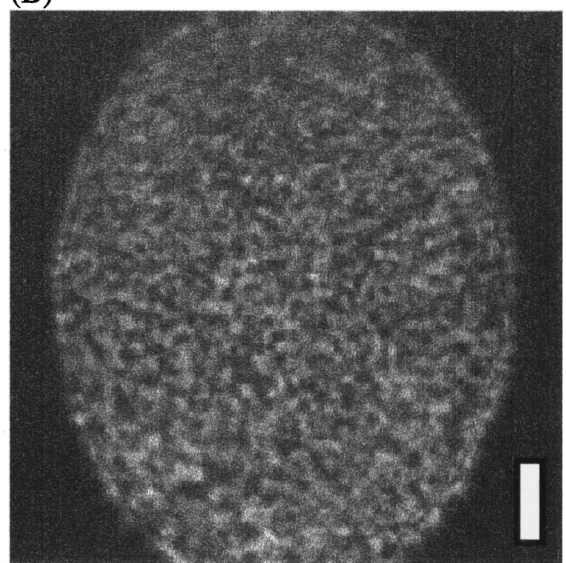

(D)

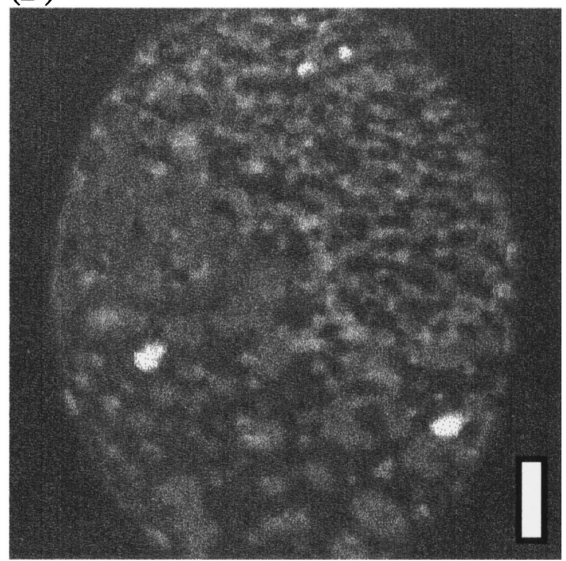

(F)

FIG. 6. BAM images of the transition from the $I^{\prime}\left(L_{2^{\prime \prime}}\right)$ phase to the $I\left(L_{2}\right)$ phase. Images (A), (C), and (E) correspond to the $s$-polarized light reflected from the monolayer, while images (B), (D), and (F) represent $p$-polarized light. Frames (A) and (B) show the film in the uncompressed state $(0$ dynes $/ \mathrm{cm})$ at $2.1{ }^{\circ} \mathrm{C}$ while in the $I^{\prime}\left(L_{2^{\prime \prime}}\right)$ phase. The polarization dependence observed in these images is indicative of a tilted phase. Note the presence of several "holes" with no polarization dependence of the reflected light [dark regions in frame (A)] in the uncompressed monolayer. In addition, the domains making up the film [see (B) ] are small, and the powder is very fine. (C) and (D) show the film while still in the $I^{\prime}\left(L_{2^{\prime \prime}}\right)$ after a slow compression to 2.3 dynes/cm and heating to $4.7^{\circ} \mathrm{C}$. The holes observed in the uncompressed film have disappeared, mainly as a result of the compression, but the polydomain texture of the sample is still very fine. Furthermore, several point like, brightly reflecting three-dimensional microcrystallites have appeared within the monolayer phase. These crystallites are observed to be strongly absorbing for $p$-polarized light [note the "disappearance" of the bright crystallites between (C) and (D)]. (E) and (F) show the monolayer after heating the film to $6.1{ }^{\circ} \mathrm{C}$. The transition to the $I\left(L_{2}\right)$ phase has taken place as observed by (1) the considerably coarser nature of the polydomain texture for the sample, (2) the larger size of the brightly reflecting three-dimensional microcrystallites, and (3) quantitative birefringence changes (Ref. 35). The white bar indicates a length scale of $100 \mu \mathrm{m}$.

ward from a presumed triple point, which is not located in Fig. 4, at the top of the $F\left(L_{2}^{\prime}\right)$ region. In this study, such a phase boundary has not been identified and although this entire region is referred to here as being occupied by the
$U(C S)$ phase, the existence of the $U^{\prime}(S)$ phase would not be contested if it were to be shown to exist in relaxed films. The x-ray area of the $U(C S)$ phase observed in this work is relatively incompressible, with $A_{X}=18.8 \pm 0.2 \AA^{2} / \mathrm{mol}$. 


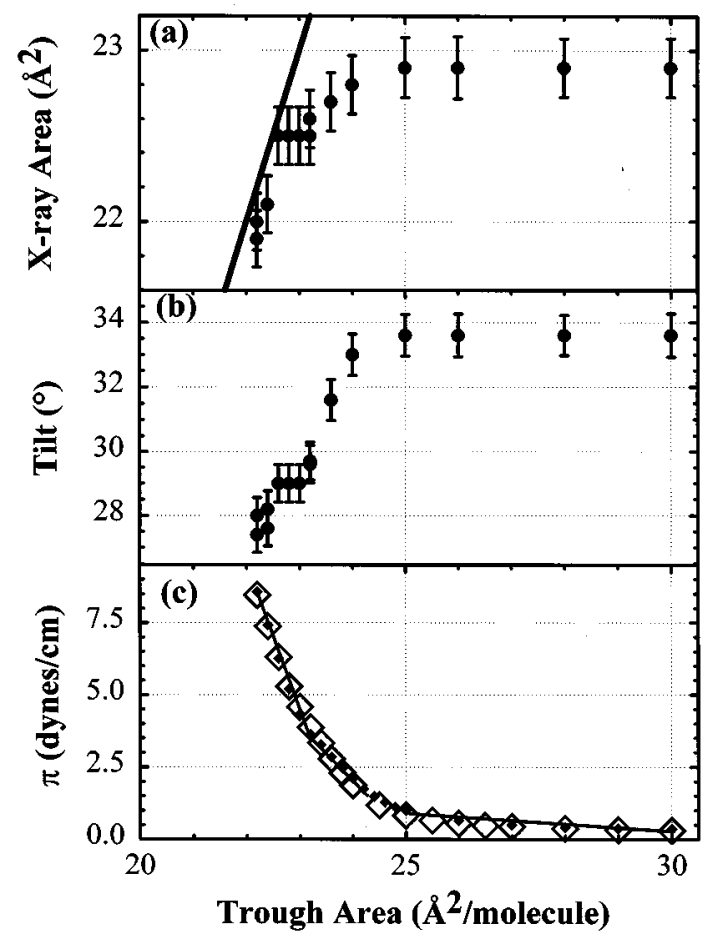

FIG. 7. Plot of the x-ray area $A_{X}$, the molecular tilt, and the surface pressure $\pi$ versus the trough area $A_{T}$, for a relaxation isotherm in the $I\left(L_{2}\right)$ phase at $20.6{ }^{\circ} \mathrm{C}$. The isotherm $[(\mathrm{c})]$ was constructed simultaneously with the diffraction measurements determining $A_{X}$ and the molecular tilt [(a) and (b)]. The solid line in (a) corresponds to $A_{X}=A_{T}$, and indicates what we would expect from a homogeneous monolayer phase. Data from two distinct films (large open and small filled diamonds) are shown in (c) in order to give some indication of the reproducibility possible in these measurements.

\section{B. Relaxation isotherms and temperature scans}

\section{Relaxation isotherms}

In this section, the manner in which the phase boundaries presented in Fig. 4 were identified through GIXS measurements during (i) isothermal compressions of a monolayer in the relaxation manner, or (ii) temperature scans at fixed $A_{T}$ will be illustrated. Although measurements from individual isotherms often display one or another anomalous feature, (see Fig. 9) from repeated measurement it was possible to distinguish the systematic and reproducible properties from anomalous features and to then verify the self-consistency of the conclusions with other experimental tools (i.e., BAM observations). The phase diagrams reported here represent the results of those efforts. In addition, details of how the respective isotherms can be interpreted to yield further information on the various phases and phase transitions will be discussed.

In Fig. 7 plots are shown for the x-ray determined molecular area, $A_{X}$; alkane chain tilt, $\theta$; and surface pressure, $\pi$; as a function of the macroscopic area per molecule deposited on the trough, $A_{T}$; for relaxation isotherms taken on two different Langmuir monolayers at $20.6{ }^{\circ} \mathrm{C}$. The isotherms [Fig. 7(c)] were measured simultaneously with the diffraction measurements determining $A_{X}$ [Fig. 7(a)] and $\theta$ [Fig. 7(b)]. The solid line in Fig. 7(a) corresponds to $A_{X}=A_{T}$, and indicates what would be expected from a homogeneous monolayer phase, for which equal changes in $A_{X}$ and $A_{T}$ are expected. Although phase transitions will generally be indicated by a change in the type of symbol used, for this figure the $I\left(L_{2}\right)$ phase is the only phase observed throughout the entire range of this measurement. Referring to Fig. 7, note that, upon spreading, the $\mathrm{x}$-ray area is smaller than the trough area. This indicates that the monolayer is not homogenous in this region of the isotherm and, as has been previously reported, it consists of islands of the ordered $I\left(L_{2}\right)$ phase in coexistence with a lower density phase. ${ }^{21,22}$ From BAM measurements, this lower density phase was observed to be a gaseous phase with immeasurably small density. The uncompressed $I\left(L_{2}\right)$ phase is characterized by a resolution limited in-plane $\left(Q_{z}=0 \AA^{-1}\right)$ GIXS peak at $Q_{x y}=1.42 \AA^{-1}$, and a degenerate out-of-plane $\left(Q_{z}=0.74 \AA^{-1}\right)$ peak at $Q_{x y}=1.40$ $\AA^{-1}$, generally broader than the resolution limit.

The in-plane diffraction peak is resolution limited, indicating that the ordered phase consists of crystalline (or hexatic) domains. Although BAM observations show islands that can be several millimeters wide, stochastic variations in Bragg intensities imply that the macroscopic islands themselves contain a mosaic distribution of $\sim 10 \mu \mathrm{m}$ crystallites separated by small angle grain boundaries. ${ }^{22,26}$ In theory, the line shape of these diffraction peaks gives information regarding the type and extent of the real-space correlations; however, the observed scattering was not strong enough to allow distinction between algebraic, Lorentzian or Gaussian line shapes. All diffraction peaks were thus fitted using a Gaussian form superimposed on a linear background to account for the bulk scattering.

Upon compression from a trough area of $30 \AA^{2} / \mathrm{mol}$, $-\left(\partial \pi / \partial A_{T}\right)_{T}$ begins to increase linearly at $A_{T} \approx 24.5 \AA^{2} / \mathrm{mol}$. However, neither $A_{X}$ nor the position of the diffraction peaks begins to change linearly with $A_{T}$, until $A_{T} \approx 23 \AA^{2} / \mathrm{mol}$. Most of the time, the out-of-plane degenerate peak for the $I\left(L_{2}\right)$ phase is not resolution limited, but the shift in its $Q_{x y}$ position is always observed to be larger than that for the in-plane peak, indicating that the lattice compresses primarily along the tilt direction. Furthermore, the $Q_{z}$ position of the out-of-plane peak decreases with decreasing trough area, implying an untilting of the monolayer. The magnitude of these changes is summarized in Fig. 7 in terms of $A_{X}$ [Fig. 7(a)] and $\theta$ [Fig. 7(b)]. At the highest pressures, the x-ray area, $A_{X}$, shrinks by approximately $1 \AA^{2} / \mathrm{mol}$ for a similar reduction in $A_{T}$, implying that in this region of the isotherm the film is homogeneous. The tilt angle systematically changes from $33^{\circ}$ to $27^{\circ}$.

For an orthorhombic symmetry, calculated values of $\theta$ that ensure closest packing of the hydrocarbon chains are: $34.5^{\circ}, 31.5^{\circ}, 27^{\circ}, 19^{\circ}$, and $0^{\circ} .{ }^{37,38}$ The uncompressed monolayer tilt angle of $33^{\circ}$ is thus reasonably close to what one would expect for a close-packed arrangement, and it is tempting to think that microscopic interchain strains associated with compression of the film may be responsible for the transitions to either the $I^{\prime}\left(L_{2^{\prime \prime}}\right)$ or $F\left(L_{2^{\prime}}\right)$ phases. Data to be shown below support this hypothesis. Nevertheless, it is also necessary to take into account thermal effects. For example, from the representation of the phase diagram in terms of $\pi$ 


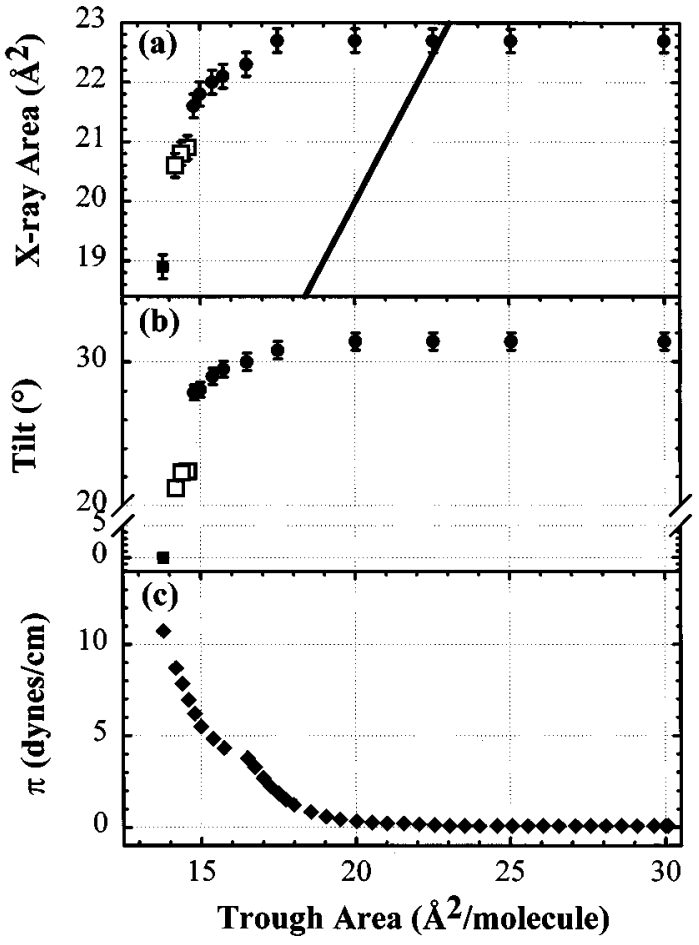

FIG. 8. X-ray and thermodynamic data summarizing the relaxation isotherm taken at $6.7^{\circ} \mathrm{C}$. The $I\left(L_{2}\right)$ (filled circles) to $F\left(L_{2}\right)$ (open squares) to $U(C S)$ (filled squares) phase transitions are shown on (a) and (b). Note that the each transition involves a discontinuous change in both $A_{X}$ and the tilt angle. Furthermore, the transitions are abrupt with no evidence for a coexistence region. Finally, note that the $I\left(L_{2}\right)$ to $F\left(L_{2}{ }^{\prime}\right)$ transition occurs well beyond the observed change in compressibility observed at $A_{T}=16.4 \AA^{2} / \mathrm{mol}$ in (c). The solid line in (a) corresponds to $A_{X}=A_{T}$, and indicates what we would expect from a homogeneous monolayer phase.

and $\theta$ (see Fig. 14 below) one can see that although $\theta$ for the transition from $I^{\prime}\left(L_{2^{\prime \prime}}\right)$ to $F\left(L_{2^{\prime}}\right)$ and $L^{\prime}\left(L_{2^{\prime \prime}}\right)$ to $I\left(L_{2}\right)$ depends on $\pi$, the slopes, $d \pi / d \theta$, within the $I\left(L_{2}\right)$ and $F\left(L_{2^{\prime}}\right)$ single phase regions are independent of temperature (within the error bars of the measurement, $\pm 0.5^{\circ}$ ), with the limiting $\theta$ value for the $I\left(L_{2}\right)$ phase varying from about $29.5^{\circ}$ at 0 dynes $/ \mathrm{cm}$ to $26.5^{\circ}$ at 11 dynes $/ \mathrm{cm}$. In addition, above $\pi=2$ dynes/cm (where the ordered portion of the monolayer is observed to be homogeneous), the change $\Delta \theta$ across the $I\left(L_{2}\right) / F\left(L_{2^{\prime}}\right)$ and $I\left(L_{2}\right) / I^{\prime}\left(L_{2^{\prime \prime}}\right)$ coexistence regions is nearly independent of $\pi$, varying from $4^{\circ} \pm 0.5^{\circ}$ at 2 dynes $/ \mathrm{cm}$ to $3^{\circ} \pm 0.5^{\circ}$ at 11 dynes $/ \mathrm{cm}$.

Finally, it is important to note the discontinuous change in the macroscopic compressibility [i.e., a change in $\left(\partial \pi / \partial A_{T}\right)_{T}$ ] of the monolayer at $A_{T}=23 \AA^{2} / \mathrm{mol}$ [see Fig. $7(\mathrm{c})]$, and the similar feature that was previously noted in the relaxation isotherm in Fig. 3. A commonly invoked explanation of features like this, when observed in constant compression isotherms, is that the change implies a phase transformation. However, such a transition is not reflected in the $\mathrm{x}$-ray measurements, where the monolayer is observed to remain in the $I\left(L_{2}\right)$ phase. What is observed is that $A_{X}$ appears to be unchanged for $\Delta A_{T} \approx 1 \AA^{2} / \mathrm{mol}$. The effect is barely within the error bars, however, the data is highly suggestive. A more likely interpretation is that the monolayer is not per-

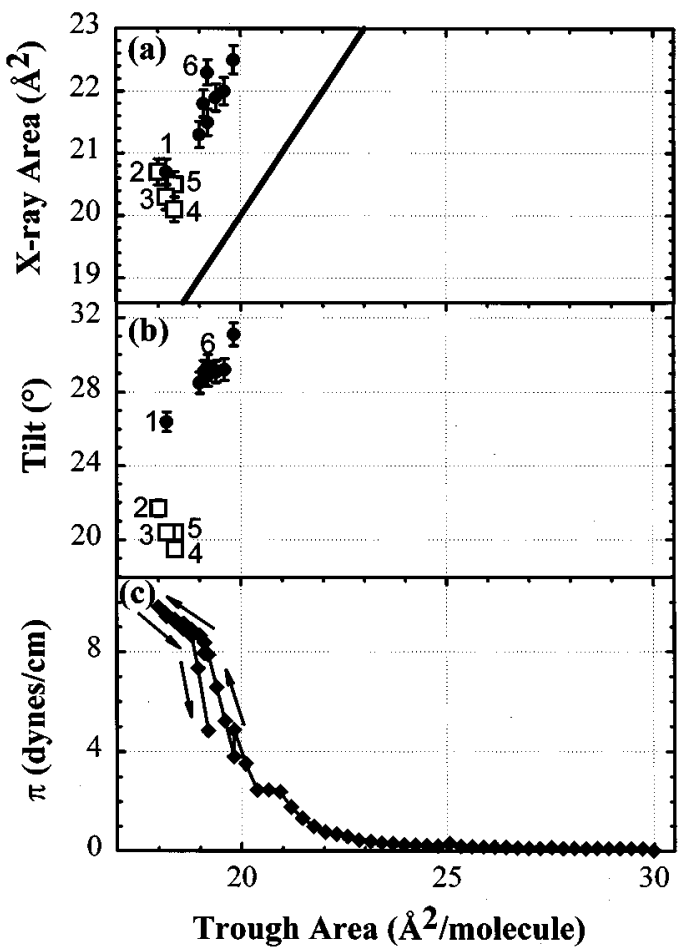

FIG. 9. Another example of the $I\left(L_{2}\right)$ (filled circles) to $F\left(L_{2}^{\prime}\right)$ (open squares) phase transition as observed upon isothermal compression of a monolayer at $8.8^{\circ} \mathrm{C}$. The transition occurs between the points labeled 1 and 2 on (a) and (b). Note that the change in $A_{X}$ involved across this transition is negligible, but the change in tilt angle is discontinuous. Once again we observe the transition well beyond the change in compressibility seen at $19.0 \AA^{2} / \mathrm{mol}$. Points labeled 3-6 were measured upon expansion of the film. Note that the $\pi-A$ isotherm exhibits some hysteresis upon expansion, and that the $F\left(L_{2}{ }^{\prime}\right)$ phase persist to $A_{T}$ values larger than $18 \AA^{2} / \mathrm{mol}$. The transition back to the $I\left(L_{2}\right)$ phase (point 5 to point 6) coincides well with the change in compressibility observed at $19 \AA^{2} /$ mol. Once again, the solid line in panel (a) corresponds to $A_{X}=A_{T}$, and indicates what we would expect from a homogeneous monolayer phase.

fectly homogeneous and at this temperature, $\pi \approx 3.5$ dynes/cm is sufficient to squeeze out defects, voids, or other imperfections in the monolayer. It follows from this, that the macroscopic pressure $\pi$ for the compressed monolayer is not precisely equal to the intrinsic thermodynamic pressure. However, by always taking data in the same manner, allowing time for relaxation effects to be completed, it is believed that such effects have been minimized.

Referring to the phase diagram (Fig. 4), the transition between the $I(L 2)$ and $F\left(L_{2^{\prime}}\right)$ phases is observed between $5.0^{\circ} \mathrm{C}$ and $12{ }^{\circ} \mathrm{C}$. Figure 8 summarizes the changes in $A_{X}, \theta$ and $\pi$ as a function of $A_{T}$ for a relaxation isotherm taken at $6.7^{\circ} \mathrm{C}$. The monolayer spreads to form the $I\left(L_{2}\right)$ phase. Once again it is noted that the initial $A_{X}$ is smaller than $A_{T}$. Thus, the measurement starts in a coexistence between the $I\left(L_{2}\right)$ phase and a dilute gas phase, just as in the room temperature measurement discussed above. The initial value of $A_{X} \approx 22.7 \AA^{2} / \mathrm{mol}$ and $\theta \approx 32^{\circ}$ are in close agreement with those observed at $20.6{ }^{\circ} \mathrm{C}$, and this indicates a very small thermal coefficient of expansion. Compression from $A_{T}=30$ $\AA^{2} / \mathrm{mol}$ to $18 \AA^{2} / \mathrm{mol}$ does not change either $A_{X}$ or $\theta$. For $A_{T} \leqslant 18 \AA^{2} / \mathrm{mol}$ the pressure begins to rise, concurrently the 
X-ray area begins to decrease. Note that at this point in the isotherm, $A_{T}$ is 4 to $5 \AA^{2} / \mathrm{mol}$ smaller than the more realistic measured values of $A_{X}$. Furthermore, the small value of $A_{T}$ is not physically realistic and it is indicative of the uncertainties that accompany attempts to develop a phase diagram in terms of $A_{T}$. Withstanding this discrepancy between the values of $A_{T}$ and $A_{X}$, while in the $I\left(L_{2}\right)$ phase the monolayer attains a homogeneous state in which changes in $A_{T}$ are reflected by equal changes in $A_{X}$.

At $A_{T}=16.4 \AA^{2} / \mathrm{mol}$ and $\pi \approx 4$ dynes $/ \mathrm{cm}$ the $\pi-A_{T}$ [Fig. $8(\mathrm{c})]$ isotherm exhibits a clear discontinuous change of compressibility. This feature was repeatedly observed on other isotherms and, without the benefit of simultaneous GIXS measurements, such features have commonly been interpreted as indicating the onset of a phase transformation. However, the x-ray data shows that the monolayer persists in the $I\left(L_{2}\right)$ phase for approximately $1.5 \AA^{2} /$ mol further compression. More importantly, the true area per molecule for the $I\left(L_{2}\right)$ phase at this transition is $A_{X}=21.6 \AA^{2} / \mathrm{mol}$, changing discontinuously to $A_{X}=20.9 \AA^{2} / \mathrm{mol}$ once in the $F\left(L_{2}^{\prime}\right)$ phase, indicating a first order phase transition. At this point, it is important to note that, as we will see throughout the rest of this paper, all phase transitions we observed lacked coexistence regions where both phases were observed simultaneously by GIXS. Thus, in this work, we identify first order phase transitions as those transitions exhibiting a discontinuous change of $A_{X}$.

The diffraction peaks characterizing the $F\left(L_{2}^{\prime}\right)$ phase (see Table I), were generally not resolution limited, and were both observed to be out-of-plane $\left(Q_{z}=0.29 \AA^{-1}\right.$ and 0.60 $\AA^{-1}$ ), indicating that the tilt order had changed from pointing toward the nearest neighbor to pointing toward the nextnearest neighbor. Also note, from Fig. 4, 13, and 14 below, that with decreasing $\pi$ the phase transition from the $I\left(L_{2}\right)$ to $I^{\prime}\left(L_{2^{\prime \prime}}\right)$ phase replaces the transition from $I\left(L_{2}\right)$ to $F\left(L_{2^{\prime}}\right)$ right after the triple point at which $\theta_{I^{\prime}}=\theta_{F} \approx 26^{\circ}$. In addition, note that the lowest values of $\theta$ for which the $F\left(L_{2^{\prime}}\right)$ phase is stable (see Fig. 14) is close to the value of $19^{\circ}$ which would indicate closest packing of the hydrocarbon chains. This is another point that must be considered in examination of the idea previously mentioned that internal strains in the chain-chain packing may be responsible for these structural transitions.

Between 9 and 10 dynes/cm, a discontinuous transition to the $U(C S)$ phase is observed by the appearance of two resolution limited peaks, corresponding to zero tilt angle and $A_{X}=18.9 \AA^{2} / \mathrm{mol}$. Following initial relaxation of $\pi$, both the surface pressure and the x-ray structure of the $U(C S)$ and $F\left(L_{2}^{\prime}\right)$ phases were stable for periods in excess of 12 hours.

Even with the anomalous features already discussed, isotherms like those shown in Fig. 8 were reproducibly observed. On the other hand, on very rare occasions, isotherms with different features which are not reproducible, like the one shown in Fig. 9 taken at $8.8^{\circ} \mathrm{C}$ are observed [note that on Fig. 12(a) we display data corresponding to an isotherm also taken at $8.8^{\circ} \mathrm{C}$, that does not exhibit the anomalous features we discuss next for the data shown in Fig. 9]. Once again there is a significant offset between the data and the

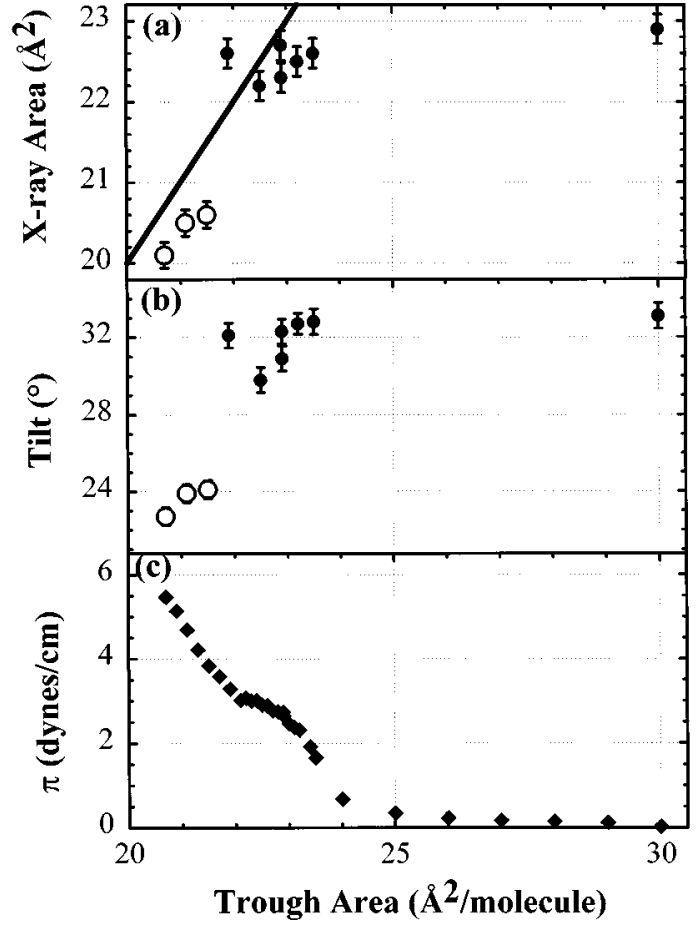

FIG. 10. X-ray and thermodynamic data summarizing the relaxation isotherm at $4.7^{\circ} \mathrm{C}$. The $I^{\prime}\left(L_{2^{\prime \prime}}\right)$ (open circles) to $I\left(L_{2}\right)$ (filled circles) phase transition is shown on (a) and (b). The transition involves an abrupt, discontinuous change in both $A_{X}$ and the tilt angle, with no evidence for a coexistence region. Finally, note that the transition occurs at $A_{T}=21.9 \AA^{2} / \mathrm{mol}$, well beyond the observed change in compressibility at $A_{T}=23 \AA^{2} / \mathrm{mol}$.

solid line, $A_{X}=A_{T}$. This might have been partially due to the region in the $\pi-A_{T}$ isotherm [Fig. 9(c)] at $A_{T} \approx 20$ to 21 $\AA^{2} /$ mol where a short plateau is observed. The value of $\pi$ at which this occurs is similar to the values of the anomalous features noted in regard to Fig. 3, 7, and 8, and the same interpretation is most likely. Once again, this does not seem to correspond to a phase transition. On further compression incremental changes $\Delta A_{T}$ are matched by equal changes in $\Delta A_{X}$. Further increases in $\pi$ yield compressibility characteristic of the $I\left(L_{2}\right)$ phase. The unlabeled filled in points in Figs. 9(a) and 9(b) represent data taken on compression. The remaining points are labeled in the order in which they were recorded. The kink at $A_{T}=19 \AA^{2} / \mathrm{mol}$ and $8.5 \mathrm{dynes} / \mathrm{cm}$ is reproducibly observed on $\pi-A_{T}$ isotherms measured about this temperature and could be taken to indicate the onset of a phase transformation. However, according to the x-ray data, the $I\left(L_{2}\right)$ to $F\left(L_{2}^{\prime}\right)$ phase transition takes place between points marked 1 and 2 , at $A_{T}=18 \AA^{2} / \mathrm{mol}\left(A_{X}=20.7 \AA^{2} / \mathrm{mol}\right)$ and 9.8 dynes $/ \mathrm{cm}$. The $I\left(L_{2}\right)$ to $F\left(L_{2}^{\prime}\right)$ transition is sharp, with a negligible change in $A_{X}$, but a discontinuous change in $\theta$ from $26.8^{\circ}$ to $21.6^{\circ}$. Upon expansion to $A_{T}>18.0$ $\AA^{2} / \mathrm{mol}$, the $F\left(L_{2}{ }^{\prime}\right)$ phase persists until $A_{T}$ is increased to 19 $\AA^{2} / \mathrm{mol}$, and $\pi$ drops to $4.8 \mathrm{dynes} / \mathrm{cm}$, at which point the $I\left(L_{2}\right)$ phase reforms (point marked 6 on Fig. 9). Hysteresis was reproducibly observed on expansion whenever a monolayer was compressed to a trough area beyond the high pressure kink seen in the isotherm. Expansion initiated prior to 


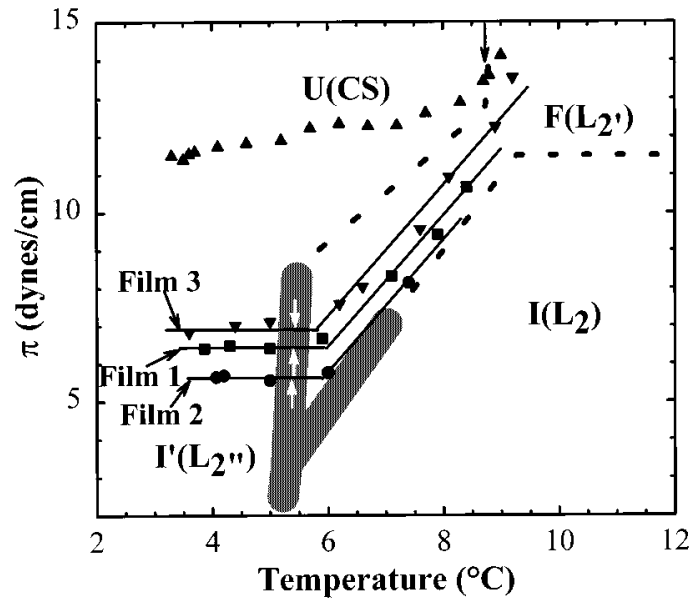

FIG. 11. Summary plot of the temperature scans discussed in this section. All of the scans represented on this figure were collected as a function of increasing temperature. The thermodynamic data extracted from the three temperature scans labeled Film 1, 2, and 3 is summarized in Table II. The initial surface pressure in each of these scans was achieved by compressing the film as in a relaxation isotherm. The arrows indicate where the phase transition was observed, and the solid lines are only a guide to the eye.

reaching this feature did not result in any hysteresis.

Referring to Fig. 9, a further anomaly can be pointed out. The smallest value of $A_{X}$ observed in the $I\left(L_{2}\right)$ phase on compression $\left(20.7 \AA^{2} / \mathrm{mol}\right)$ is approximately $0.7 \AA^{2} / \mathrm{mol}$ smaller than expected when compared with numerous other values measured over a range of temperatures and surface pressures [see the $\pi-A_{X}$ phase diagram displayed below in Fig. 13, where the $\left(\pi, A_{X}\right)$ point at 10.2 dynes $/ \mathrm{cm}$ and 20.7 $\AA^{2} / \mathrm{mol}$ is shown as an $\left.X\right]$. It is believed that for this particular monolayer the $I\left(L_{2}\right)$ phase is supercompressed to approximately $\delta A_{X} \approx 0.7 \AA^{2} / \mathrm{mol}$ smaller area than the equilibrium phase boundary.

Data for the $I\left(L_{2}\right)$ to $I^{\prime}\left(L_{2^{\prime \prime}}\right)$ phase transition as observed on an isotherm taken at $4.7^{\circ} \mathrm{C}$ are illustrated in Fig. 10. Upon spreading, the film is found once again to be inhomogeneous. Once the pressure begins to increase, $A_{X}$ starts to decrease proportionally to changes in $A_{T}$. Starting at $A_{T} \approx 23 \AA^{2} / \mathrm{mol}$ and 2.4 dynes $/ \mathrm{cm}$, the onset of a plateau in the $\pi-A_{T}$ isotherm is observed. Once again, such features are commonly interpreted as the onset of a first order phase transition with coexistence of the two phases. However, the x-ray data only shows a sharp phase transition to the $I^{\prime}\left(L_{2^{\prime \prime}}\right)$ phase occurring at the end of this plateau $\left(A_{T}=21.9 \AA^{2} / \mathrm{mol}\right)$, over a change in area $\Delta A_{T} \leqslant 0.2 \AA^{2} / \mathrm{mol}$, where the pressure is beginning to rise. Generally, both the in-plane and out-ofplane $\left(Q_{z}=0.52 \AA^{-1}\right)$ diffraction peaks characteristic of the $I^{\prime}\left(L_{2^{\prime \prime}}\right)$ phase were found to be resolution limited. Once again we point out that no coexistence of $I\left(L_{2}\right)$ and $I^{\prime}\left(L_{2^{\prime \prime}}\right)$ phases was observed by GIXS. The change in $A_{X}$ across the phase transition is approximately $2 \AA^{2} / \mathrm{mol}$, which correlates well with the width of the plateau on the $\pi-A_{T}$ isotherm and the values for $\theta$ change discontinuously from $32^{\circ}$ to $24^{\circ}$. The $I\left(L_{2}\right)$ phase is stable for many hours in the plateau region.
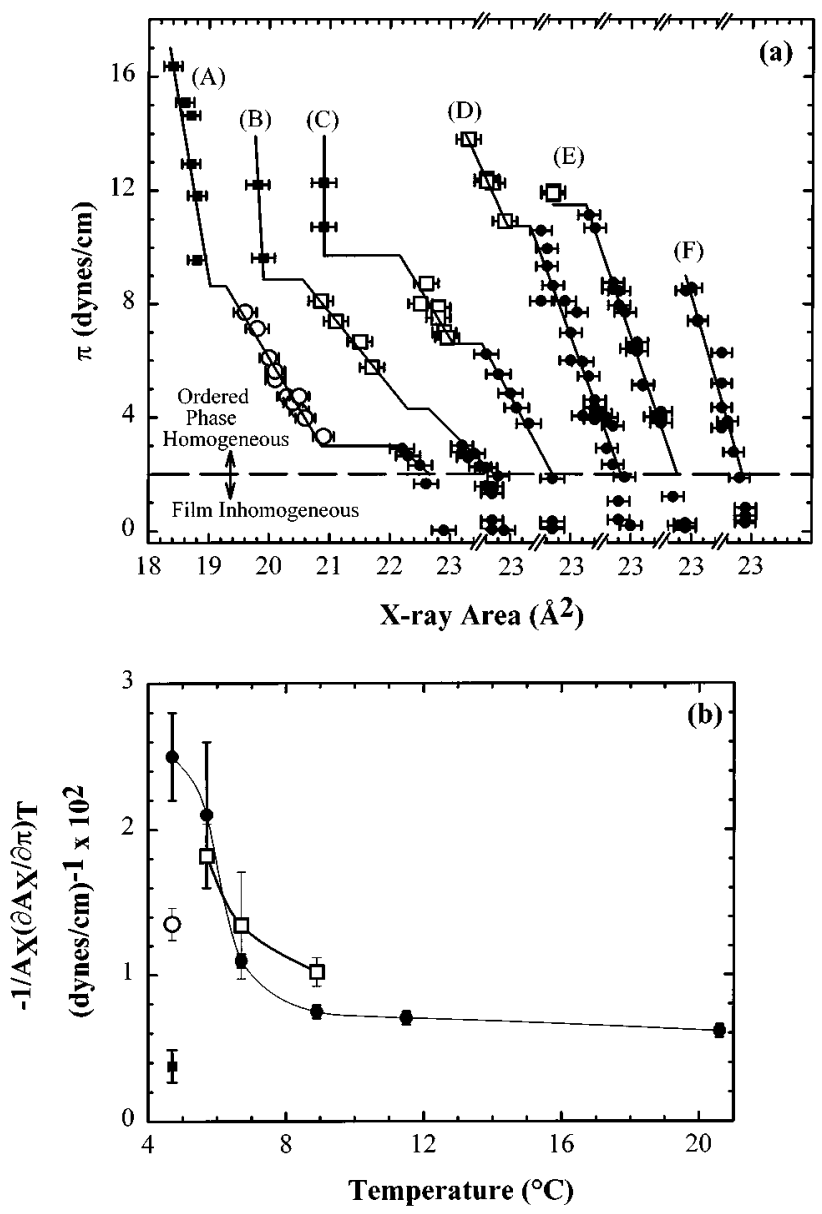

FIG. 12. (a) Plot of several isotherms on the $\pi-A_{X}$ plane. Phases are identifiable as follows: the $I\left(L_{2}\right)$ phase is shown as filled circles, the $I^{\prime}\left(L_{2^{\prime \prime}}\right)$ phase appears as open circles, the open squares represent the $F\left(L_{2}^{\prime}\right)$ phase, and the $U(C S)$ phase is depicted using filled squares. The sequence of temperatures corresponding to the isotherms shown is as follows: (A) $4.7^{\circ} \mathrm{C}$, (B) $5.7^{\circ} \mathrm{C}$, (C) $6.7^{\circ} \mathrm{C}$, (D) $8.8^{\circ} \mathrm{C}$, (E) $11.5^{\circ} \mathrm{C}$, and (F) $20.6{ }^{\circ} \mathrm{C}$. The solid lines represent linear fits to the data, except in cases where two or less points for a given phase on a given isotherm were present. (b) The compressibility for each phase, extracted from the linear fits of the data displayed in (a), is plotted as a function of temperature. Phases in this figure are identified using the same symbols used in (a).

\section{Temperature scans (isochores)}

Referring to Fig. 4, the phase transition between the $I^{\prime}\left(L_{2^{\prime \prime}}\right)$ and $F\left(L_{2}\right)$ phases is nearly isothermal and the temperature is not stable enough that the transition can be characterized by a relaxation isotherm of the type shown in Fig. 10. Consequently, this transition was characterized by temperature scans, in which the trough area is held fixed while the surface pressure changes as a function of changing temperature. Since the macroscopic density is held fixed, it would be appropriate to refer to these measurements as isochores. However, although the macroscopic density (i.e., $A_{T}$ ) is fixed, the x-ray area $A_{X}$ changes as a function of temperature, and to avoid any confusion that could arise because of this we have purposely chosen to refer to this set of measurements simply as temperature scans. The particular series of temperature scans presented here all started from the lowest temperature attainable in our system $\left(\sim 3{ }^{\circ} \mathrm{C}\right)$, below the 
$I\left(L_{2}\right) / I^{\prime}\left(L_{2^{\prime \prime}}\right)$ boundary, insuring that spreading occurs in the $I^{\prime}\left(L_{2^{\prime \prime}}\right)$ phase. This is important because relaxation isotherms of a film spread in the $I^{\prime}\left(L_{2^{\prime \prime}}\right)$ phase do not show the anomalous features that were reported in discussing isotherms of monolayers spread in the $I\left(L_{2}\right)$ phase. After spreading, the film was compressed just as in a relaxation isotherm to the surface pressure at which the temperature scan was initiated. The temperature was typically changed in steps of $1-2{ }^{\circ} \mathrm{C}$, allowing for both temperature and surface pressure to come to steady state values before making any $\mathrm{x}$-ray measurements.

Figure 11 shows a compilation of the temperature scans at fixed $A_{T}$ (connected symbols), plotted against the phase boundaries that were shown in Fig. 4. The single most important feature consistently observed is a sharp change in the slope of the $\pi$ vs temperature trace immediately following the point where GIXS indicated a phase transition from $I^{\prime}\left(L_{2^{\prime \prime}}\right)$ to $F\left(L_{2^{\prime}}\right)$ (shown by the arrows on Fig. 11). It is also important to note that the location of the phase transition does not depend strongly on the initial $\pi$ over the range explored by these data $(5-7$ dynes $/ \mathrm{cm})$, indicating that the coexistence curve does not possess pronounced curvature in this pressure range. Combining the temperature scan data and the data recorded on isothermal compressions close to the $I^{\prime}\left(L_{2^{\prime \prime}}\right) / I\left(L_{2}\right) / F\left(L_{2^{\prime}}\right)$ triple point, a lower limit on the slope of the $I^{\prime}\left(L_{2^{\prime \prime}}\right) / F\left(L_{2^{\prime}}\right)$ coexistence line can be set at $d \pi / d T=17 \pm 6$ dynes $/ \mathrm{cm}-{ }^{\circ} \mathrm{C}$.

\section{The $\pi-A_{X}$ phase diagram}

A principal point of this manuscript, alluded to in the previous discussion, is that the occurrence of anomalies in $\pi-A_{T}$ isotherms make the $\pi-A_{T}$ plane a poor choice for representation of the Langmuir monolayer phase diagram. We suspect that the anomalies are either due to disordered regions that our $\mathrm{x}$-ray measurements do not probe, or due to other effects such as 3D crystallites that form outside the sample area exposed to the $\mathrm{x}$ rays. These regions affect the compressibility or other properties of the monolayer and can be responsible for kinks or other anomalous features. The thermodynamic role of these regions in influencing the phase behavior of the ordered parts of the monolayer is unclear; however, by allowing the monolayer to relax, a consistent thermodynamic description of the behavior of the ordered regions of the monolayer emerges. This description is best represented in the $\pi-A_{X}$ plane which explicitly excludes regions that our x-ray measurements do not probe.

Isotherms plotted in the $\pi-A_{X}$ plane enable the determination of the compressibility of the ordered phases, and the extent to which transitions are continuous or discontinuous in $A_{X}$. This is illustrated in Fig. 12(a), where a series of isotherms is plotted in terms of $\pi$ vs $A_{X}$. Referring to this figure, the compressibility $-1 / A_{X}\left(\partial A_{X} / \partial \pi\right)_{T}$ of the $I\left(L_{2}\right)$ phase determined for $\pi>2$ dynes/cm (where the ordered phase is found to be homogeneous), is observed to be temperature dependent [see Fig. 12(b)]; varying nonlinearly from $0.61 \times 10^{-2} \pm 0.04 \times 10^{-2}$ (dynes/cm) ${ }^{-1}$ at $20.6^{\circ} \mathrm{C}$ to a maximum of $2.5 \times 10^{-2} \pm 0.3 \times 10^{-2}(\text { dynes } / \mathrm{cm})^{-1}$ at $4.7^{\circ} \mathrm{C}$.
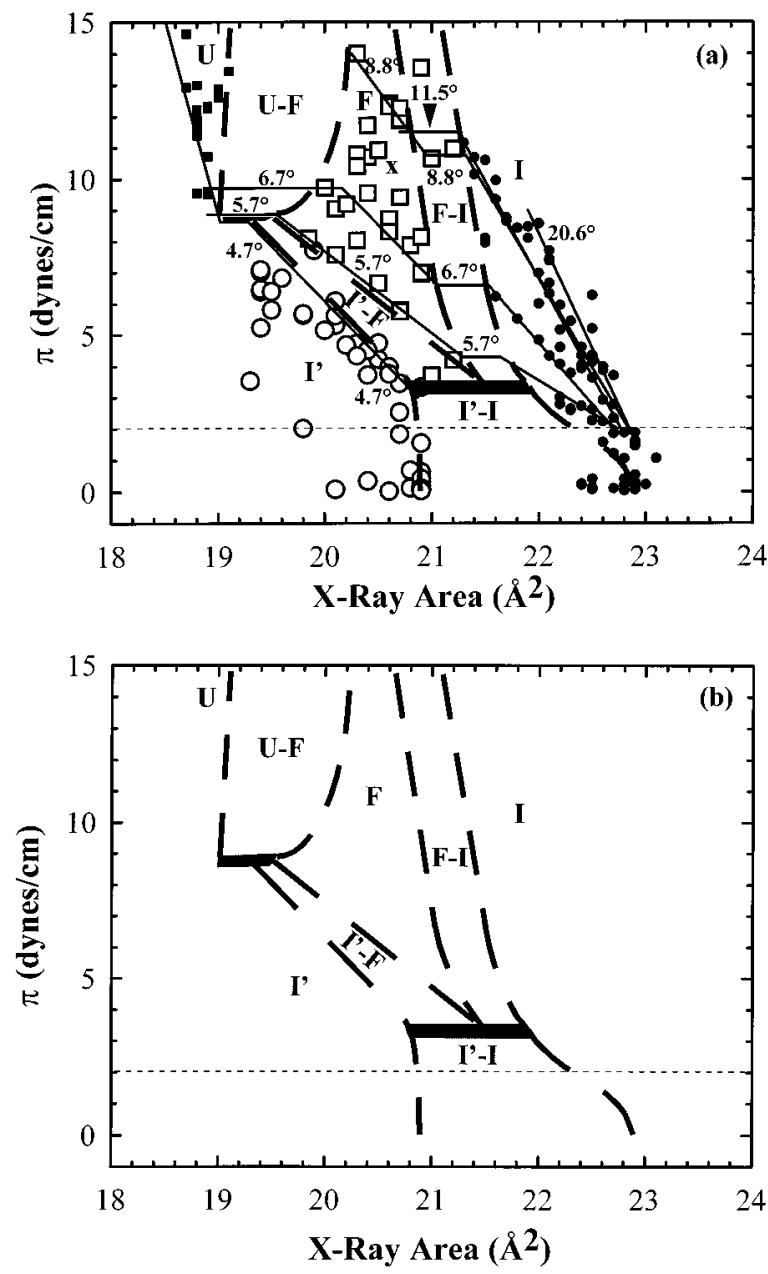

FIG. 13. (a) Surface pressure versus x-ray area phase diagram. Each symbol represents an individual $x$-ray measurement. Phases are identifiable as follows: the $I\left(L_{2}\right)$ phase is shown as filled circles, the $I^{\prime}\left(L_{2^{\prime \prime}}\right)$ phase appears as open circles, the open squares represent the $F\left(L_{2}^{\prime}\right)$ phase, and the $U(C S)$ phase is depicted using filled squares. Solid lines labeled with a temperature identify a series of isotherms. Thick dashed lines are used to mark phase boundaries and identify coexistence regions. Note that on this phase diagram, the $I^{\prime}\left(L_{2^{\prime \prime}}\right) / I\left(L_{2}\right) / F\left(L_{2^{\prime}}\right)$ and the upper triple points are represented by horizontal shaded areas, centered at 3.5 and 8.9 dynes $/ \mathrm{cm}$, respectively. The thin horizontal dashed line at $\pi=2$ dynes $/ \mathrm{cm}$ indicates the following: below this pressure the Langmuir film is observed to be inhomogeneous, whereas above this pressure the film is observed to be homogeneous. (b) For added clarity, this figure reproduces only the phase boundaries observed in the surface pressure $\pi$ vs x-ray area phase diagram presented in (a).

Similarly, $-1 / A_{X}\left(\partial A_{X} / \partial \pi\right)_{T}$ for the $F\left(L_{2^{\prime}}\right)$ phase is also observed to depend on temperature, increasing from $1.02 \times 10^{-2} \pm 0.10 \times 10^{-2} \quad(\text { dynes } / \mathrm{cm})^{-1}$ at $8.8^{\circ} \mathrm{C}$ to $1.82 \times 10^{-2} \pm 0.22 \times 10^{-2}$ (dynes $\left./ \mathrm{cm}\right)^{-1}$ at $5.7^{\circ} \mathrm{C}$. Finally, the compressibility for the $U(C S)$ and $I^{\prime}\left(L_{2^{\prime \prime}}\right)$ at $4.7^{\circ} \mathrm{C}$ were measured to be $0.38 \times 10^{-2} \pm 0.11 \times 10^{-2}$ (dynes $\left./ \mathrm{cm}\right)^{-1}$ and $1.35 \times 10^{-2} \pm 0.11 \times 10^{-2}$ (dynes/cm) ${ }^{-1}$, respectively. Unfortunately, not enough data was collected for these two phases to determine the temperature dependence of their compressibility. At the lowest temperatures (below $6{ }^{\circ} \mathrm{C}$ ), where a measurement of $-1 / A_{X}\left(\partial A_{X} / \partial \pi\right)_{T}$ was made for all four phases, one finds the compressibility to decrease in the order: $I\left(L_{2}\right)>F\left(L_{2^{\prime}}\right)>I^{\prime}\left(L_{2^{\prime \prime}}\right)>U(C S)$. Since the packing ar- 

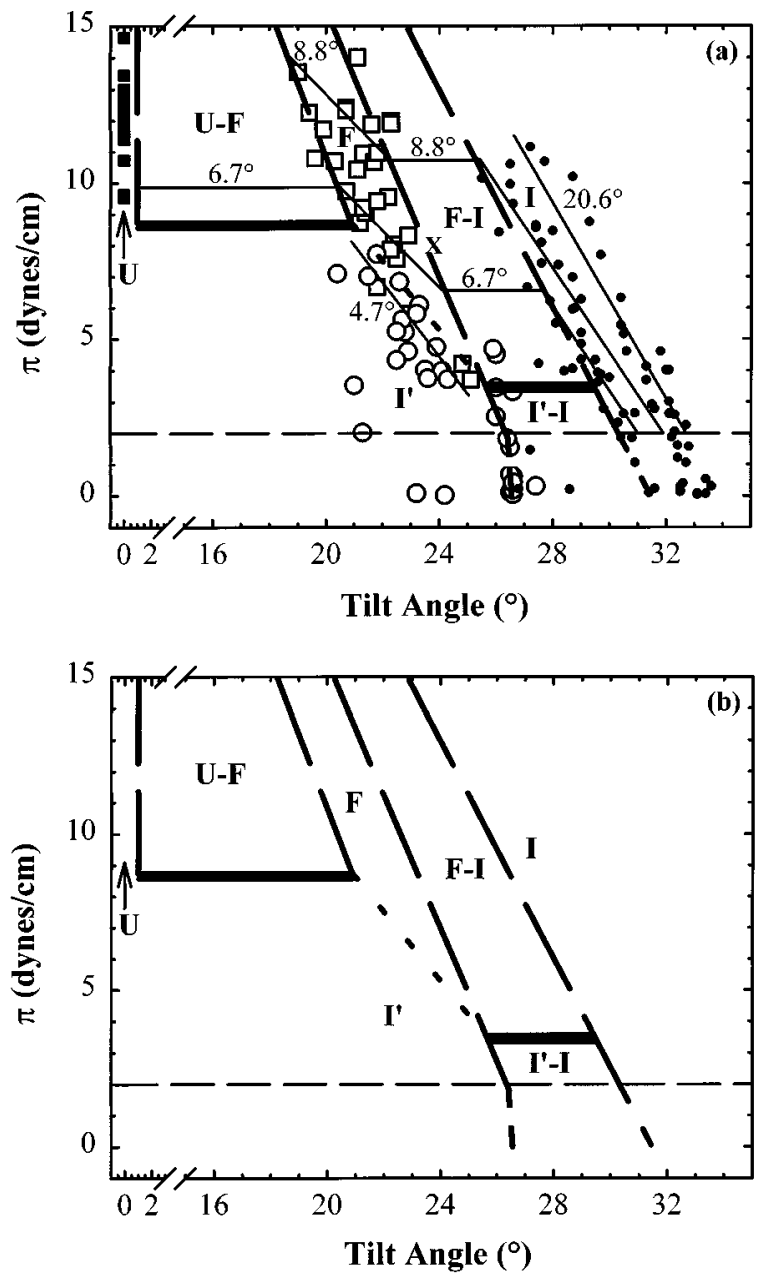

FIG. 14. (a) Surface pressure vs tilt angle phase diagram. Each symbol represents an individual $\mathrm{x}$-ray measurement. Phases are identifiable as follows: the $I\left(L_{2}\right)$ phase is shown as filled circles, the $I^{\prime}\left(L_{2}^{\prime \prime}\right)$ phase appears as open circles, the open squares represent the $F\left(L_{2^{\prime}}\right)$ phase, and the $U(C S)$ phase is depicted using filled squares. Dashed lines are used to mark phase boundaries and identify coexistence regions. Solid lines labeled with a temperature identify a series of isotherms. Horizontal thick solid lines indicate the location of the $I^{\prime}\left(L_{2^{\prime \prime}}\right) / I\left(L_{2}\right) / F\left(L_{2^{\prime}}\right)$ triple point at $3.5 \mathrm{dynes} / \mathrm{cm}$ and the upper triple point at 8.9 dynes $/ \mathrm{cm}$. The thick dotted line indicates the $I^{\prime}\left(L_{2^{\prime \prime}}\right) / F\left(L_{2^{\prime}}\right)$ phase boundary. Note that although the $I^{\prime}\left(L_{2^{\prime \prime}}\right) / F\left(L_{2^{\prime}}\right)$ phase transition is continuous with respect to $\theta$, it involves a change in the tilt order from $\mathrm{NN}$ to NNN. The thin horizontal dashed line at $\pi=2$ dynes/cm indicates the following: below this pressure the Langmuir film is observed to be inhomogeneous, whereas above this pressure the film is observed to be homogeneous. (b) For added clarity, this figure reproduces only the phase boundaries observed in the surface pressure $\pi$ vs tilt angle phase diagram presented in (a).

rangement for both the $I^{\prime}\left(L_{2^{\prime \prime}}\right)$ and $U(C S)$ phases is observed to be the two-dimensional analog of a stable polymorphic fatty acid bulk crystalline phase, ${ }^{38}$ these phases should be relatively incompressible. Similarly, a bulk analog is not found for the $I\left(L_{2}\right)$ phase, and its larger compressibility is consistent with the observation that, in this region (below $\left.6{ }^{\circ} \mathrm{C}\right)$, the diffraction peaks characterizing the $I\left(L_{2}\right)$ phase are not resolution limited. Thus, the order of compressibility's reported here seems to be consistent with these observations, and it appears that one can indeed use isotherms plot- ted on the $\pi-A_{X}$ plane to determine the compressibility of ordered phases in Langmuir monolayers.

Figure 13 shows the phase diagram of Behenic acid as a function of $\pi-A_{X}$. Each point represents an individual GIXS measurement and the different phases are represented by the same symbols that were used in Fig. 4. The solid lines labeled with temperatures identify a series of isotherms, and coexistence regions are defined using thicker dashed lines. Our location for the $I^{\prime}\left(L_{2^{\prime \prime}}\right) / I\left(L_{2}\right) / F\left(L_{2^{\prime}}\right)$ and upper triple points (see Fig. 4) are represented on the diagram in Fig. 13 by horizontal shaded areas centered at approximately 3.5 and 8.7 dynes $/ \mathrm{cm}$. The location of the $I^{\prime}\left(L_{2^{\prime \prime}}\right) / I\left(L_{2}\right) / F\left(L_{2^{\prime}}\right)$ triple point is based on the Clausius-Clapeyron relation discussed below.

The $I\left(L_{2}\right)$ phase exists in a narrow region of the phase diagram, never more than $\approx 1 \AA^{2} / \mathrm{mol}$ wide for a given temperature, covering a range of $A_{X}$ from $23 \AA^{2} / \mathrm{mol}$ to 21.2 $\AA^{2} / \mathrm{mol}$ and extending from 0 dynes $/ \mathrm{cm}$ to 11 dynes $/ \mathrm{cm}$. Below $3.5 \mathrm{dynes} / \mathrm{cm}$ and at smaller $A_{X}$, there is a coexistence region between the $I\left(L_{2}\right)$ and the $I^{\prime}\left(L_{2^{\prime \prime}}\right)$ phases. Within the homogeneous region, the width of this coexistence varies, from $\Delta A_{X} \sim 1.5 \AA^{2} / \mathrm{mol}$ at 2 dynes $/ \mathrm{cm}$ to $1.1 \AA^{2} / \mathrm{mol}$ at the $I^{\prime}\left(L_{2^{\prime \prime}}\right) / I\left(L_{2}\right) / F\left(L_{2^{\prime}}\right)$ triple point, reflecting mostly the compressibility of the $I\left(L_{2}\right)$ phase. Above 3.5 dynes $/ \mathrm{cm}$, there is an $I\left(L_{2}\right)-F\left(L_{2}^{\prime}\right)$ coexistence which is about 0.5 $\AA^{2} /$ mol wide at the $I^{\prime}\left(L_{2^{\prime \prime}}\right) / I\left(L_{2}\right) / F\left(L_{2^{\prime}}\right)$ triple point, and remains constant to 11.5 dynes $/ \mathrm{cm}$. As mentioned earlier, although the $I\left(L_{2}\right)$ to $F\left(L_{2^{\prime}}\right)$ transition observed in the isotherm shown in Fig. 9 appears to occur with $\Delta A_{X} \approx 0$, it is believed to have been an anomalous observation in which the $I\left(L_{2}\right)$ phase persisted past the normal phase boundary.

The $I^{\prime}\left(L_{2^{\prime \prime}}\right)$ phase has an intermediate $A_{X}$ and is located at low pressures, while the $F\left(L_{2^{\prime}}\right)$ phase is observed at intermediate $A_{X}$ and high pressures. The $I^{\prime}\left(L_{2^{\prime \prime}}\right)-F\left(L_{2^{\prime}}\right)$ coexistence region bridges these two phases. Even though the slope of the $I^{\prime}\left(L_{2^{\prime \prime}}\right)-F\left(L_{2^{\prime}}\right)$ coexistence line is a very steep function of temperature (see Fig. 4), it encompasses a fairly large range in $A_{X}$ (Fig. 13).

The $U(C S)$ phase appears at small $A_{X}$ and high pressures. There is a clear coexistence with the $F\left(L_{2^{\prime}}\right)$ phase, and the width of this coexistence varies with respect to pressure. It can be speculated that the change in $A_{X}$ across the $I^{\prime}\left(L_{2^{\prime \prime}}\right)$ to $U(C S)$ phase transition may be quite small, and is likely to be zero, but further measurements would be necessary to substantiate this conclusion.

Finally, to complete this discussion, the $\pi-A_{X}$ phase diagram, recast in terms of $\pi$ vs $\theta$, is presented in Fig. 14. Discontinuous changes in the magnitude of $\theta$ are observed for all phase transitions except the $I^{\prime}\left(L_{2^{\prime \prime}}\right)$ to $F\left(L_{2^{\prime}}\right)$ transition which entails only a change in the tilt order from NN to NNN. The $I\left(L_{2}\right)$ to $F\left(L_{2^{\prime}}\right)$ and $I\left(L_{2}\right)$ to $I^{\prime}\left(L_{2^{\prime \prime}}\right)$ transitions involve an average change in tilt angle of $\sim 3.5 \pm 0.5^{\circ}$. As mentioned previously, this change is constant (within error bars) as a function of pressure, for both the $I^{\prime}\left(L_{2^{\prime \prime}}\right)-I\left(L_{2}\right)$ and $I\left(L_{2}\right)-F\left(L_{2^{\prime}}\right)$ coexistence regions. Contrary to the suggestion that phase transitions occur once particular values of $\theta$ are achieved, it is clear from this diagram that there is a temperature dependent range of $\theta$ over which the $I\left(L_{2}\right)$ 
TABLE II. Summary of thermodynamic parameters extracted from temperature scans about the $I^{\prime}\left(L_{2^{\prime \prime}}\right) / F\left(L_{2^{\prime}}\right)$ transition.

\begin{tabular}{|c|c|c|c|c|c|}
\hline Film & Phase & $\begin{array}{c}T \text { range } \\
\left({ }^{\circ} \mathrm{C}\right)\end{array}$ & $\begin{array}{c}(\partial \pi / \partial T)_{N, A_{T}} \\
\left(\text { dynes } / \mathrm{cm}-{ }^{\circ} \mathrm{C}\right)\end{array}$ & $\begin{array}{c}A_{x} \\
\left(\AA^{2} / \mathrm{mol}\right)\end{array}$ & $\Delta S_{I^{\prime} F}\left(k_{B}\right)^{\mathrm{a}}$ \\
\hline Film 1 & $\begin{array}{l}I^{\prime}\left(L_{2^{\prime \prime}}\right) \\
F\left(L_{2^{\prime}}\right)\end{array}$ & $\begin{array}{l}3.9-5.8 \\
5.8-8.4\end{array}$ & $\begin{array}{l}-0.14 \pm 0.04 \\
-1.69 \pm 0.14\end{array}$ & $\begin{array}{l}19.3 \pm 0.2 \\
20.5 \pm 0.2\end{array}$ & $15 \pm 5$ \\
\hline Film 2 & $\begin{array}{l}I^{\prime}\left(L_{2^{\prime \prime}}\right) \\
F\left(L_{2^{\prime}}\right)\end{array}$ & $\begin{array}{l}4.1-6.0 \\
6.0-7.4\end{array}$ & $\begin{array}{l}-0.19 \pm 0.06 \\
-1.84^{\mathrm{b}}\end{array}$ & $\begin{array}{l}19.5 \pm 0.2 \\
20.6 \pm 0.2\end{array}$ & $14 \pm 4$ \\
\hline Film 3 & $\begin{array}{l}I^{\prime}\left(L_{2^{\prime \prime}}\right) \\
F\left(L_{2^{\prime}}\right)\end{array}$ & $\begin{array}{l}3.6-6.4 \\
6.4-9.2\end{array}$ & $\begin{array}{l}-0.34 \pm 0.02 \\
-1.72 \pm 0.17\end{array}$ & $\begin{array}{l}19.4 \pm 0.2 \\
20.6 \pm 0.2\end{array}$ & $15 \pm 5$ \\
\hline Averages & $\begin{array}{l}I^{\prime}\left(L_{2^{\prime \prime}}\right) \\
F\left(L_{2^{\prime}}\right)\end{array}$ & & $\begin{array}{l}-0.22 \pm 0.03 \\
-1.75 \pm 0.11\end{array}$ & $\begin{array}{l}19.4 \pm 0.1 \\
20.6 \pm 0.1\end{array}$ & $15 \pm 5$ \\
\hline
\end{tabular}

${ }^{\mathrm{a}}$ Calculated using a value of $d \pi_{I^{\prime} F} / d T=17 \pm 6$ dynes $/ \mathrm{cm}-{ }^{\circ} \mathrm{C}$.

${ }^{\mathrm{b}}$ This slope is determined by only two data points.

phase can transform to either the $I^{\prime}\left(L_{2^{\prime \prime}}\right)$ or $F\left(L_{2^{\prime}}\right)$ phases. In other words, it does not appear that there are only discrete values of $\theta$ for which the $I\left(L_{2}\right)$ phase becomes unstable and undergoes a transformation. In view of the fact that $\theta$ and molecular density are strongly coupled, the values of $\theta$ at which a phase transition occurs should be a continuous function of density. For a system with finite compressibility, this implies that $\theta$ is a continuous function of $\pi$, as is seen in Fig. 14 . With this caveat in mind, it is worth noting that the range of $\theta$ achievable by the $I\left(L_{2}\right)$ phase is from $32.5^{\circ}$ to $26.5^{\circ}$, and no stable $I\left(L_{2}\right)$ phase observed for $\theta<26.5^{\circ}$. Similarly, both the $I^{\prime}\left(L_{2^{\prime \prime}}\right)$ and $F\left(L_{2^{\prime}}\right)$ phases can be stable to $\theta$ as small as $19^{\circ}-20^{\circ}$, but not smaller. These observations are consistent with the calculated sequence of $\theta$ ensuring close of the hydrocarbon chains of $34.5^{\circ}, 31.5^{\circ}, 27^{\circ}, 19^{\circ}$, and $0^{\circ} .{ }^{37,38}$ The $U(C S)$ to $F\left(L_{2^{\prime}}\right)$ transition also involves discontinuous changes in $\theta$.

\section{DISCUSSION AND INTERPRETATION}

\section{A. Thermodynamics and transition entropies}

Assuming that the phase diagram shown in Fig. 4 is reasonably close to the thermodynamic equilibrium diagram, the Clausius-Clapeyron equation for a monolayer,

$$
\frac{d \pi_{I^{\prime} F}}{d T}=\frac{\Delta S_{I^{\prime} F}}{\left(\Delta A_{X}\right)_{I^{\prime} F}}
$$

can be used to calculate the change in entropy associated with the $I^{\prime}\left(L_{2^{\prime \prime}}\right)$ to $F\left(L_{2^{\prime}}\right)$ transition: $d \pi_{I^{\prime} F} / d T$ is the slope of $I^{\prime}\left(L_{2^{\prime \prime}}\right) / F\left(L_{2^{\prime}}\right)$ phase boundary on a $\pi-T$ phase diagram, $\Delta S_{I^{\prime} F}$ is the entropy change associated with the phase transition, and $\left(\Delta A_{X}\right)_{I^{\prime} F}=\left(A_{X}\right)_{I^{\prime}}-\left(A_{X}\right)_{F}$ is the difference in the X-ray area of the $I^{\prime}\left(L_{2^{\prime \prime}}\right)$ and $F\left(L_{2^{\prime}}\right)$ phases, respectively. Note that practical application of the Clausius-Clapeyron equation is only possible because $\left(\Delta A_{X}\right)_{I^{\prime} F}$ is directly measured by GIXS. The thermodynamic data regarding this transition is summarized in Table II and was obtained from the three different temperature scans at fixed $A_{T}$ that were started from $\pi=5.7,6.5$, and 7.0 dynes $/ \mathrm{cm}$ with the film in the $I^{\prime}\left(L_{2^{\prime \prime}}\right)$ phase (see Fig. 11). Listed in Table II for each film are the temperature range over which the phases were followed, the slopes $(\partial \pi / \partial T)_{N, A_{T^{\prime}}}$ and the $A_{X}$ prior to, and following, the transition. The last column lists the values for the lower limit on $\Delta S_{I^{\prime} F}$ [in units of Boltzmann's constant $\left(k_{B}\right)$ ] calculated using Eq. (1) and the lower limit on the slope of the coexistence line $\left(d \pi_{I^{\prime} F} / d T=17 \pm 6\right.$ dynes $/ \mathrm{cm}$ $\left.{ }^{\circ} \mathrm{C}\right)$ for the boundary between the $I^{\prime}\left(L_{2^{\prime \prime}}\right)$ and $F\left(L_{2}\right)$ phases. The average value of $\Delta S_{I^{\prime} F}$ was found to be $15 \pm 5 \mathrm{kB}$. Similarly, from the slope of the $F\left(L_{2^{\prime}}\right) / I\left(L_{2}\right)$ boundary measured between $6{ }^{\circ} \mathrm{C}$ and $9{ }^{\circ} \mathrm{C}, d \pi_{F I} / d T=1.9 \pm 0.2$ dynes $/ \mathrm{cm}-{ }^{\circ} \mathrm{C}$, and the difference $\left(A_{X}\right)_{I}-\left(A_{X}\right)_{F}=0.5 \pm 0.1 \AA^{2} / \mathrm{mol}$, the value of $\Delta S_{F I}$ is calculated to be $2.7 \pm 0.3 k_{B}$.

If we assume all the three phase boundaries are straight

TABLE III. Summary of thermodynamic parameters for several bulk systems. $\Delta S_{P}$ is the change in entropy per molecule for a polymorphic transition (i.e., from a rotator or a crystalline phase to a crystalline phase), while $\Delta S_{M}$ is the entropy change measured for solidification (i.e., from the liquid phase to a rotator or crystalline phase). The $\Delta S$ quantities were calculated from the measured latent heats $(\Delta H)$ of the given transition. The corresponding transition temperatures are also given.

\begin{tabular}{|c|c|c|c|c|c|c|}
\hline System & $\begin{array}{c}\Delta H_{P} \\
(\mathrm{~kJ} / \mathrm{mole})\end{array}$ & $T_{P}\left({ }^{\circ} \mathrm{C}\right)$ & $\Delta S_{P}\left(k_{B}\right)$ & $\begin{array}{c}\Delta H_{M} \\
(\mathrm{~kJ} / \mathrm{mole})\end{array}$ & $T_{M}\left({ }^{\circ} \mathrm{C}\right)$ & $\Delta S_{M}\left(k_{B}\right)$ \\
\hline Behenic Acid ${ }^{\mathrm{b}} \mathrm{C}_{22} \mathrm{H}_{44} \mathrm{O}_{2}$ & $\cdots$ & $\ldots$ & $\ldots$ & 78.7 & 80.0 & 26.8 \\
\hline Tricosanoic Acid ${ }^{\mathrm{b}} \mathrm{C}_{23} \mathrm{H}_{46} \mathrm{O}_{2}$ & 6.8 & 77.5 & $2.3^{\mathrm{a}}$ & 73.6 & 79.1 & 25.2 \\
\hline Docosane ${ }^{\mathrm{b}} \mathrm{C}_{22} \mathrm{H}_{46}$ & 28.2 & 43.0 & $10.7^{\mathrm{a}}$ & 49.0 & 44.4 & 18.5 \\
\hline Docosanol $^{\mathrm{b}} \mathrm{C}_{22} \mathrm{H}_{46} \mathrm{O}$ & $\cdots$ & $\cdots$ & $\cdots$ & 91.2 & 70.0 & 32.0 \\
\hline
\end{tabular}

${ }^{a}$ Values calculated using a molecular area of $20 \AA^{2}$.

${ }^{\mathrm{b}}$ Reference 37. 
TABLE IV. Summary of thermodynamic parameters for several surface systems. (a) The first column identifies the bulk liquid system, the second and third columns give the temperature derivative of the surface pressure $d \pi / d T=-d \gamma / d T$ prior to and following surface freezing, and the fourth column shows the change $(\Delta S)$ in entropy across this surface transition, assuming a molecular area of $20 \AA^{2} / \mathrm{mol}$. The next two columns compare the surface freezing transition temperature $\left(T_{L}\right.$ to $\left.C\right)$ and the melting point of the bulk material $\left(T_{M}\right)$. (b) The thermodynamic data from ESP measurements of fatty acids is listed in the same manner as that from surface tension measurements of bulk alkanes and alcohols.

(a) Surfaces of neat systems

\begin{tabular}{|c|c|c|c|c|c|}
\hline System & $\begin{array}{c}(d \gamma / d T)_{L} \\
\left(\text { dynes } / \mathrm{cm}-{ }^{\circ} \mathrm{C}\right)\end{array}$ & $\begin{array}{c}(d \gamma / d T)_{C} \\
\left(\text { dynes } / \mathrm{cm}-{ }^{\circ} \mathrm{C}\right)\end{array}$ & $\begin{array}{l}\Delta S_{L} \text { to } C \\
\quad\left(k_{B}\right)\end{array}$ & $\begin{array}{l}T_{L \text { to } C} C \\
\left({ }^{\circ} \mathrm{C}\right)\end{array}$ & $\begin{array}{c}T_{M} \\
\left({ }^{\circ} \mathrm{C}\right)\end{array}$ \\
\hline Docosane ${ }^{\mathrm{a}} \mathrm{C}_{22} \mathrm{H}_{46}$ & -0.09 & 1.72 & $26.2^{\mathrm{b}}$ & & \\
\hline Eicosane $^{a} \mathrm{C}_{20} \mathrm{H}_{40}$ & -0.09 & 1.32 & $20.4^{\mathrm{b}}$ & 38.6 & 36.8 \\
\hline Octadecane ${ }^{\mathrm{a}} \mathrm{C}_{18} \mathrm{H}_{36}$ & -0.09 & 0.91 & $14.5^{\mathrm{b}}$ & 30.6 & 28.2 \\
\hline Docosanol $^{\mathrm{c}} \mathrm{C}_{22} \mathrm{H}_{46} \mathrm{O}$ & -0.10 & 1.82 & $27.8^{\mathrm{b}}$ & 70.0 & 69.3 \\
\hline Eicosanol $^{\mathrm{c}} \mathrm{C}_{20} \mathrm{H}_{40} \mathrm{O}$ & -0.11 & 1.40 & $21.9^{\mathrm{b}}$ & 64.7 & 64.0 \\
\hline Octadecanol $^{c} \mathrm{C}_{18} \mathrm{H}_{36} \mathrm{O}$ & -0.09 & 1.37 & $21.2^{\mathrm{b}}$ & 57.6 & 56.9 \\
\hline
\end{tabular}

(b) Equilibrium spreading pressure of surfactants

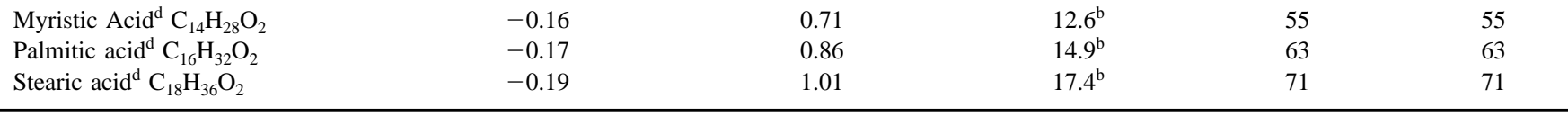

${ }^{\mathrm{a}}$ Reference 39.

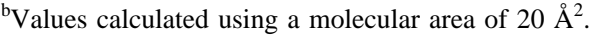

${ }^{\mathrm{c}}$ Reference 40 .

${ }^{\mathrm{d}}$ Reference 43.

lines approaching the $I^{\prime}\left(L_{2^{\prime \prime}}\right) / I\left(L_{2}\right) / F\left(L_{2^{\prime}}\right)$ triple point, and $\Delta S_{I^{\prime} F}+\Delta S_{F I} \approx \Delta S_{I^{\prime} I}=17.7 k_{B}$, from the measured difference $\left(A_{X}\right)_{I^{-}}-\left(A_{X}\right)_{I^{\prime}} \approx 1.1 \pm 0.2 \AA^{2} / \mathrm{mol}$ the slope of the $I^{\prime}\left(L_{2^{\prime \prime}}\right)$ to $I\left(L_{2}\right)$ coexistence line in the vicinity of the multiple point is predicted to be $22 \pm 12$ dynes $/ \mathrm{cm}-{ }^{\circ} \mathrm{C}$. The $I^{\prime}\left(L_{2^{\prime \prime}}\right)$ to $I\left(L_{2}\right)$ phase boundary and the location of the triple point shown in Fig. 4 are obtained by drawing a line with this slope through the intersection of the straight lines for the $I^{\prime}\left(L_{2^{\prime \prime}}\right) / F\left(L_{2^{\prime}}\right)$ and $I\left(L_{2}\right) / F\left(L_{2^{\prime}}\right)$ boundaries. It is important to note that the location of the $I^{\prime}\left(L_{2^{\prime \prime}}\right) / I\left(L_{2}\right)$ boundary thus determined is in agreement with the location of this boundary as identified using GIXS data from temperature scans at 0 dynes $/ \mathrm{cm}$.

The entropy associated with the $I^{\prime}\left(L_{2^{\prime \prime}}\right)$ to $F\left(L_{2^{\prime}}\right)$ and the $I^{\prime}\left(L_{2^{\prime \prime}}\right)$ to $I\left(L_{2}\right)$ transitions calculated here can also be compared to related values in similar bulk and surface systems. Tables III and IV summarize the measured entropy changes associated with specified phase transitions for several bulk and surface systems. Table III lists thermodynamic data for polymorphic (where available) and melting phase transitions for long chain fatty acids, alkanes, and alcohols. ${ }^{37}$ The entropy change, $\Delta S$, is calculated from the measured latent heats $(\Delta H)$ of the transition. The subscript $P$ identifies polymorphic transitions while the subscript $M$ identifies crystal melting transitions.

Thermodynamic data from recent surface tension, $\gamma$, measurements of a surface freezing transition for bulk samples of normal alkane and alcohol liquids is presented in Table IV. ${ }^{39-41}$ In these studies, just as in our temperature scans, the thermodynamic signature for the surface transition is a discontinuous change in $d \gamma / d T$ while scanning temperature. This transition is clearly distinguished from the bulk transition by the fact that the surface transition temperature is greater than for the bulk transition. GIXS measurements con- firm that these surface transitions correspond to the formation of a crystalline surface layers prior to bulk solidification. ${ }^{37}$ In all cases the entropy/molecule of the bulk melting transitions is within a factor of two of the above values for the entropy of the $I^{\prime}\left(L_{2^{\prime \prime}}\right)$ to $F\left(L_{2^{\prime}}\right)$ and $I^{\prime}\left(L_{2^{\prime \prime}}\right)$ to $I\left(L_{2}\right)$ transitions for the behenic acid Langmuir monolayer. Consistent with the GIXS data, the implication of this is that both the $I^{\prime}\left(L_{2^{\prime \prime}}\right)$ to $F\left(L_{2^{\prime}}\right)$ and $I^{\prime}\left(L_{2^{\prime \prime}}\right)$ to $I\left(L_{2}\right)$ transitions involve first order melting of a well ordered $2 \mathrm{D}$ solid, or crystalline $I^{\prime}\left(L_{2^{\prime \prime}}\right)$ phase, into what is either a weakly ordered 2D crystal or a mesomorphic (i.e., 2D hexatic) phase. It should be pointed out that for bulk fatty acid liquids, surface freezing prior to bulk solidification has not been observed. ${ }^{42}$

The thermodynamic data in Table IV for the equilibrium spreading pressure (ESP) measurements of three long-chain fatty-acids indicates similar transition entropies for other Langmuir monolayers. ${ }^{43}$ ESP measurements are performed by placing a given amount of the bulk component in contact with a water subphase and measuring the surface tension of the air/water interface as a function of decreasing temperature. For the fatty acid systems listed here, ESP measurements show a kink in $d \pi / d T=-d \gamma / d T$ at precisely the melting point of the bulk fatty acid. For comparison, the thermodynamic data from these ESP measurements is listed in the same manner as that from surface tension measurements of bulk alkanes and alcohols. It is also worth noting that in contrast to fatty acid systems, the ESP measurements of short chain alcohols have shown phase transitions at temperatures considerably higher than the bulk melting point of the material. ${ }^{26,42}$ Of particular importance here is a comparison with the ESP measurements of Berge et al. ${ }^{26}$ They combined $\mathrm{x}$-ray scattering results with a thermodynamic analysis based on the Clausius-Clapeyron equation to determine the 


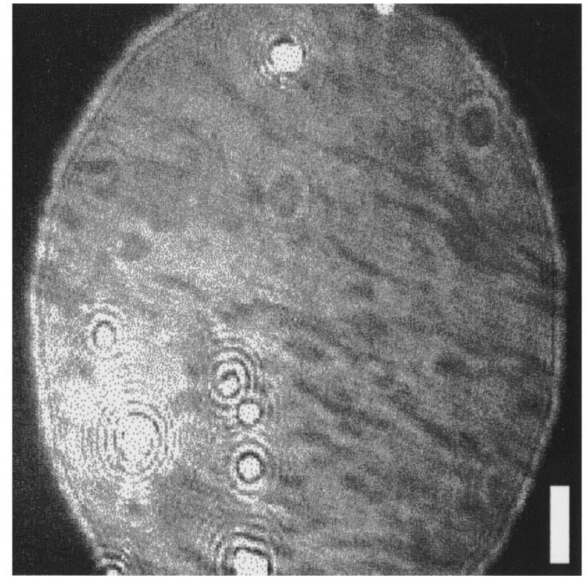

(A)

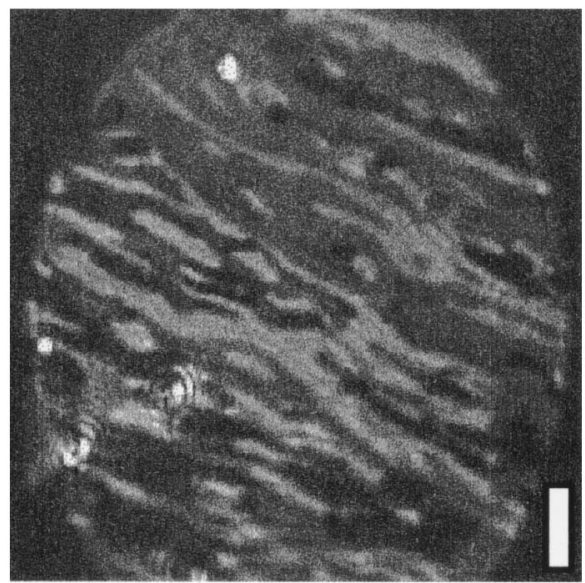

(B)

FIG. 15. (A) BAM image of a monolayer spread at $14.5^{\circ} \mathrm{C}$, in the $I\left(L_{2}\right)$ phase. The image was recorded soon after spreading while still in the uncompressed state, and represents only the $s$-polarized light reflected by the monolayer. Note the presence of several brightly reflecting point like features, interspersed amidst the two-dimensional polydomain sample representative of the $I\left(L_{2}\right)$ phase. These bright objects are probably microscopically small three dimensional crystallites. The white bar indicates a length scale of $100 \mu \mathrm{m}$. (B) Same portion of the monolayer as in (a), showing the $p$-polarized light reflected by the film. Here, the polydomain nature of the $I\left(L_{2}\right)$ phase is more clearly evident. Furthermore, note that most of the crystallites present in (a), have become strongly absorbing. The white bar indicates a length scale of $100 \mu \mathrm{m}$.

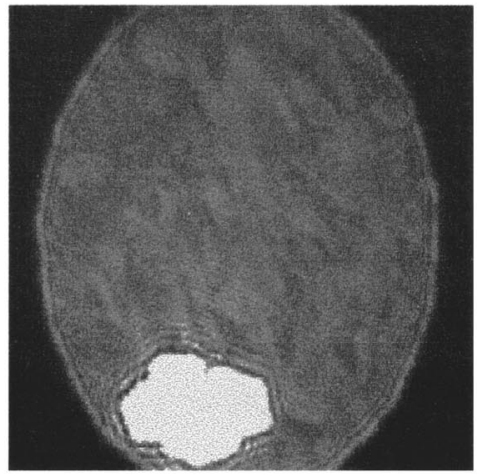

(A)

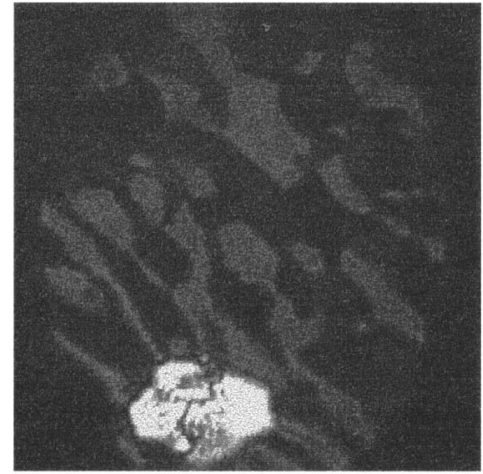

(B)

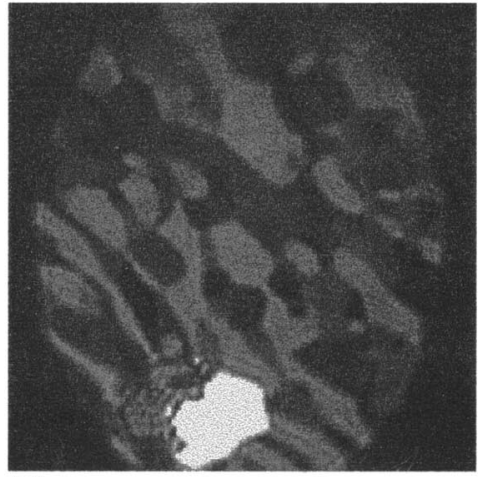

(C)

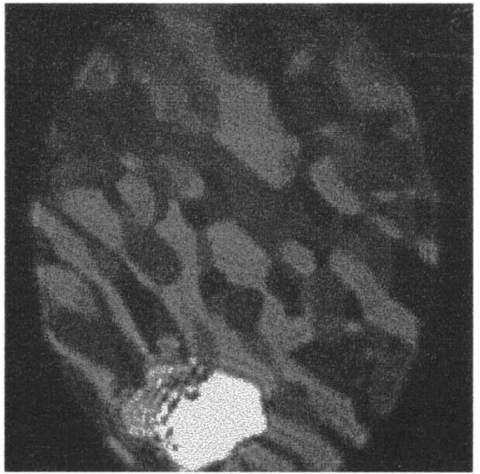

(D)

FIG. 16. BAM images of slowly compressed $\left(0.14 \AA^{2} /\right.$ molecule-hour) monolayer at $14.5^{\circ} \mathrm{C}$ and 4.3 dynes $/ \mathrm{cm}$, in the $I\left(L_{2}\right)$ phase. Frames $(\mathrm{A})$-(D) were taken with an analyzer in the path of the reflected light at $0^{\circ}$ ( $s$-polarized light), $80^{\circ}, 90^{\circ}$ ( $p$-polarized light), and $100^{\circ}$, respectively. Note that the domains making up the powder sample have grown significantly when compared to the uncompressed state of the film [see Fig. 15(a) and 15(b)]. Furthermore, the pointlike features observed in Fig. 15(a) and 15(b), have become hexagonal in shape [see cluster of crystals at bottom of frames (A)-(D)] and have grown significantly in size. In addition, while some of the crystals become strongly absorbing for $p$-polarized light, others remain strongly reflective. The white bar indicates a length scale of $100 \mu \mathrm{m}$. 
melting entropy for monolayers of short chain alcohols (chain lengths less than or equal to 16 carbons) on water, in equilibrium with a liquid lens of the bulk material. They found that the melting point of the monolayer is $15^{\circ} \mathrm{K}$ above the melting point of the bulk material, and the melting entropy $\Delta S_{M}$ increases linearly with chain length $(n)$ according to

$$
\Delta S_{M} \sim(n-8.2) k_{B} .
$$

From this equation, extrapolating to $n=22$ (where an actual measurement was not made), one obtains $\Delta S_{M}=13.8$ $k_{B}$, which is very close to the value of $\Delta S$ measured here for the $I^{\prime}\left(L_{2^{\prime \prime}}\right)$ to $F\left(L_{2^{\prime}}\right)$ and the $I^{\prime}\left(L_{2^{\prime \prime}}\right)$ to $I\left(L_{2}\right)$ phase transitions. This supports the conclusion reached above that these transitions involve first order melting.

Although there is not yet a definitive measurement capable of establishing whether the $I\left(L_{2}\right)$ and $F\left(L_{2^{\prime}}\right)$ phases are two dimensional crystals with "quasilong range", positional order ${ }^{44-47}$ or some type of hexatic phase, from the slope of the $I^{\prime}\left(L_{2^{\prime \prime}}\right) / F\left(L_{2}^{\prime}\right)$ coexistence line, and by extrapolation to the $I^{\prime}\left(L_{2^{\prime \prime}}\right) / I\left(L_{2}\right)$ coexistence line, the $I\left(L_{2}\right)$ and $F\left(L_{2}^{\prime}\right)$ phases do have a relatively large fraction of the entropy of melting for bulk behenic acid. This might suggest that these two phases are hexatic. ${ }^{48,49}$ The GIXS data does not conclusively support this suggestion, although generally the diffraction peaks corresponding to the $F\left(L_{2}{ }^{\prime}\right)$ phase, and almost always the degenerate peak of the $I\left(L_{2}\right)$ phase, were observed to be broader than the resolution limit. In contrast, the diffraction peaks for the $I^{\prime}\left(L_{2}^{\prime \prime}\right)$ phase always appeared resolution limited. Regardless of whether or not the $I\left(L_{2}\right)$ and $F\left(L_{2}^{\prime}\right)$ phases might have "quasilong range" positional order or not, the relatively small slope of the $I\left(L_{2}\right)$ to $F\left(L_{2^{\prime}}\right)$ coexistence line, for similar changes of $\Delta A_{X}$, indicates that the $F\left(L_{2}^{\prime}\right) / I\left(L_{2}\right)$ transition does not involve a large change in entropy.

\section{B. Anomalous features}

To shed some light on the possible nature of the observed discrepancies between $A_{T}$ and $A_{X}$, the texture of the relaxed films was analyzed under the BAM. In the BAM, small unresolved features that appear to have hexagonal symmetry, many times brighter than the monolayer [Figs. 15 (a) and $15(\mathrm{~b})]$ were regularly observed for monolayers spread above $\sim 5^{\circ} \mathrm{C}$, where the equilibrium monolayer phase consisted of $I\left(L_{2}\right)$ domains separated by what appeared to be the bare substrate $\left(\mathrm{H}_{2} \mathrm{O}\right)$ surface. Figures 15(a) and 15(b) illustrate the same area of the monolayer with analyzer set for $s$ and $p$ polarization, respectively. The bright objects are probably microscopically small 3D crystallites, and although their number density is very low immediately after spreading, it does increase as a function of time. In the uncompressed $I\left(L_{2}\right)$ film, the crystals remain small and unresolvable. However, when the film is compressed very slowly $\left(0.14 \AA^{2} / \mathrm{mol}-\mathrm{h}\right)$ from this initial stage, the size and number of these crystals grows. In all cases studied, when compression is carried out this slowly, the crystals develop hexagonal like facets (Fig. 16), and grow to 50-200 $\mu \mathrm{m}$ in width (on average). If compressions are carried out slightly faster $(0.3$ $\AA^{2} /$ mol-h, which is a typical compression used when collecting GIXS data), the crystallites do not grow considerably in either number density or size. Furthermore, they remain small and never develop the macroscopic hexagonal-like facets. In addition, once the monolayer has been compressed beyond a surface pressure of $\sim 3$ to 5 dynes $/ \mathrm{cm}$, no further growth of the crystallites with time is observed. Anomalous effects are more likely to be observed in monolayers which remain in the low pressure region for extended periods of time. These bright pointlike crystals are rarely seen when the monolayer is spread below $5^{\circ} \mathrm{C}$, where the isolated domains are in the $I^{\prime}\left(L_{2^{\prime \prime}}\right)$, rather than the $I\left(L_{2}\right)$ phase. Furthermore, when they are observed, their number and size do not increase perceptibly with time or compression, even when the compression rate is as small as $0.1 \AA^{2} /$ mol-h. This point is important because it correlates with the presence of anomalous features, such as kinks or plateaus, seen in $\pi-A_{T}$ isotherms of monolayers spread above $5{ }^{\circ} \mathrm{C}$ (see Fig. 9), but never observed when compressions were initiated with monolayers spread below $5^{\circ} \mathrm{C}$, in the $I^{\prime}\left(L_{2}{ }^{\prime \prime}\right)$ phase.

\section{SUMMARY}

Sections of the $\pi-T$ phase diagram of Behenic acid on water $(p \mathrm{H}=2.0, \mathrm{HCl}$ buffer $)$ have been constructed from a series of GIXS measurements collected during both isothermal compressions in which $\pi$ was allowed to fully relax, ${ }^{21,22}$ and temperature scans at fixed $A_{T}$. The four distinct phases that were observed consist of two tilted phases with $\mathrm{NN}$ tilt order $\left[I\left(L_{2}\right)\right.$ and $\left.I^{\prime}\left(L_{2^{\prime \prime}}\right)\right]$, one tilted phase with NNN tilt order $\left[F\left(L_{2}^{\prime}\right)\right]$, and an untilted phase $[U(C S)]$. Plotted on a $\pi-T$ surface (see Fig. 4), the topology of the phase diagram, below $12{ }^{\circ} \mathrm{C}$, is similar to what already has been reported in the literature for Langmuir monolayers of long chain fatty acids. ${ }^{7-13}$ However, as a consequence of allowing the surface pressure to fully relax between incremental compressions, the range of $\pi$ spanned by the present phase diagram (0 to $10-15$ dynes $/ \mathrm{cm}$ ) is considerably smaller than for most published phase diagrams ( 0 to 50-60 dynes/cm). Nevertheless, in this smaller range of $\pi$, the phase diagram presented here exhibits most of the same phases observed over the larger $\pi$ range of published diagrams. Accompanying the much lower collapse pressures exhibited by relaxed monolayers is the fact that the phase diagram in Fig. 4, over the temperature range from 3 to $12^{\circ} \mathrm{C}$, does not result from "truncating', the previously reported phase diagrams at a lower collapse pressure, but rather from "compressing', the reported diagrams into the smaller pressure range. In other words, for the phase diagram built from relaxation isotherms for $T<12{ }^{\circ} \mathrm{C}$, all of the phases observable are exhibited over a narrower pressure range than in the case of diagrams constructed based on constant compression isotherms. When the temperature increases above $12^{\circ} \mathrm{C}$, the phase diagram presented here actually lacks some of the phases, such as the rotator phases, observed at large $\pi$ (i.e., when $\pi>10-15$ dynes $/ \mathrm{cm}$, above the typical collapse pressure of a relaxed monolayer) in the published phase diagrams.

In addition, the present study reiterates previous 
observations ${ }^{21,22}$ that in the more compressed portions of the phase diagram, where GIXS measurements indicate the monolayer to be in ordered two-dimensional crystalline or hexatic phases, there are frequent discrepancies between $A_{T}$ and $A_{X}$. In view of the fact that $A_{X}$ is obviously a good intensive variable for the ordered phase, it is difficult to understand how $A_{T}$ can also be an acceptable intensive thermodynamic variable of the system. Indeed, when the phases observed are displayed on a $\pi-A_{X}$ phase diagram, the resulting diagram is highly satisfactory. In fact, with the data displayed in this way, the elementary thermodynamic arguments contained in the Clausius-Clapeyron relation can be invoked to locate the $I^{\prime}\left(L_{2^{\prime \prime}}\right) / I\left(L_{2}\right)$ phase boundary (in agreement with GIXS measurements) and to determine measured values for the transition entropy between different monolayer phases.

Independent BAM studies ${ }^{50}$ have shown that growth of microcrystallites similar to what was observed here is not observed for the alcohol analog of $C_{22}$ (docosanol), either upon spreading or as a result of compression. Similarly, although crystallites are not observed for Langmuir monolayers of $C_{16}$ fatty acid (hexadecanoic acid) at temperatures higher than $20^{\circ} \mathrm{C}$ they are observed for $\mathrm{C}_{18}$ fatty acid ${ }^{50}$ (stearic acid) spread from solution at $5.3{ }^{\circ} \mathrm{C}$. These observations correlate well with what is known regarding the spreading behavior of these materials. The $C_{22}$ and $C_{18}$ fatty acids do not spread from the bulk (at least in the temperature range of interest here), while both the $C_{22}$ alcohol (as reported in the literature ${ }^{17}$ ) and the $C_{16}$ fatty acid (for subphase temperatures greater than $20^{\circ} \mathrm{C}$ ) spread from the bulk to give measurable equilibrium spreading pressures. A conclusion can be drawn that materials observed to spread spontaneously from the bulk phase will possess a range of surface pressures (below the equilibrium spreading pressure) for which the 2D monolayer phases are truly at equilibrium. On the other hand, for a given set of experimental conditions, materials that do not spread spontaneously from the bulk may spread from solution to form metastable monolayer phases from which 3D bulk crystallites will form if allowed by kinetics. This conclusion is further substantiated by empirical observation of the fact that monolayers of $C_{22}$ acid tend to exhibit more anomalous relaxation effects if they are allowed to stay in the uncompressed state longer than usual. This leads us to suggest that anomalous monolayer relaxation effects in isothermal compressions may be explained by whether or not these $3 \mathrm{D}$ crystals are present as nucleation centers. Although these type of effects add a certain degree of stochasticity to the present measurements, by systematic examination of the data and measurement conditions, consistent data sets can be extracted. The effects described above clearly are relevant in attempting to understand deposition of Langmuir-Blodgett films.

In addition to the choice of $A_{X}$ as the proper intensive variable, rather than $A_{T}$, and the use of temperature scans for characterization of the phase diagram, also important is the question of whether the internal pressure within an ordered domain and the macroscopic surface pressure measured by the Wilhelmy plate method are the same. Gradual formation of 3D crystallites does not seem to occur for condensed monolayers in the region where long relaxations are observed $(\pi \approx 4$ dynes/cm). X-Ray and BAM studies indicate that under these conditions the monolayers consist of compressed mosaics of polydomain samples. Presumably, the slow relaxations observed in the relaxation isotherms, even before collapse, originate from some form of grain boundary relaxation. Furthermore, if the individual 2D grains are elastically anisotropic, it is not obvious that the micro variations in the local stresses are negligible. Hysteresis effects, like those shown in Fig. 9, could be a consequence of just such effects.

The present studies were carried out under the assumption that by consistently making measurements where relaxations were allowed to come to completion, these effects could be minimized. The consistency of the various data sets, often taken months apart, is a partial justification for this assumption. On the other hand, these effects could be extremely relevant in materials applications, and a better understanding of exactly what determines these effects and how to control them, would then be very important. Furthermore, we have shown that temperature scans are not subject to this anomalous behavior, and allow a thermodynamically rigorous analysis of the various phase transitions to learn more about their physicochemical nature.

\section{ACKNOWLEDGMENTS}

The research reported here was supported by the Grant Nos. NSF-DMR-94-00396 and NSF-DMR-91-13782. Some experiments were carried out at the National Synchrotron Light Source, Brookhaven National Laboratory which is supported by DOE Contract No. DE-AC02-76CH00016.

${ }^{1}$ J. D. Swalen, D. L. Allara, J. D. Andrade, E. A. Chandross, S. Garoff, J. Israelachvili, T. J. McCarthy, R. Murray, and R. F. Pease, Langmuir 3, 932 (1987).

${ }^{2}$ E. M. Landau, M. Levanon, L. Leiserowitz, M. Lahav, and J. Sagiv, Nature 318, 353 (1985).

${ }^{3}$ E. M. Landau, R. Popovitz-Biro, M. Levanon, L. Leiserowitz, M. Lahav, and J. Sagiv, Mol. Cryst. Liq. Cryst. 134, 323 (1986).

${ }^{4}$ E. M. Landau, S. Grayer-Wolf, J. Sagiv, M. Deutsch, K. Kjaer, J. AlsNielsen, L. Leiserowitz, and M. Lahav, Pure Appl. Chem. 61, 673 (1989).

${ }^{5}$ J. P. deGennes and J. Prost, The Physics of Liquid Crystals (Oxford University Press, New York, 1994).

${ }^{6}$ P. S. Pershan, Structure of Liquid Crystal Phases (World Scientific, Singapore, 1988).

${ }^{7}$ S. Stallberg-Stenhagen and E. Stenhagen, Nature 156, 239 (1945).

${ }^{8}$ A. M. Bibo and I. Peterson, Adv. Mat. 2, 309 (1990).

${ }^{9}$ B. Lin, M. C. Shih, T. M. Bohanon, G. E. Ice, and P. Dutta, Phys. Rev. Lett. 65, 191 (1990).

${ }^{10}$ R. M. Kenn, C. Böhm, A. M. Bibo, I. R. Peterson, H. Möhwald, K. Kjaer, and J. Als-Nielsen, J. Phys. Chem. 95, 2092 (1991).

${ }^{11}$ A. M. Bibo, C. M. Knobler, and I. R. Peterson, J. Phys. Chem. 95, 5591 (1991).

${ }^{12}$ G. A. Overbeck and D. Mobius, J. Phys. Chem. 97, 7999 (1993).

${ }^{13}$ D. K. Schwartz and C. M. Knobler, J. Phys. Chem. 97, 8849 (1993).

${ }^{14}$ W. Rabinovitch, R. F. Robertson, and S. G. Mason, Can. J. Chem. 38, 1881 (1960)

${ }^{15}$ N. R. Pallas and B. A. Pethica, Langmuir 1, 509 (1985).

${ }^{16} \mathrm{G}$. L. Gaines, Jr., Insoluble Monolayers at Liquid-Gas Interfaces (Wiley, New York, 1966).

${ }^{17}$ K. S. Birdi, Lipid and Biopolymer Monolayers at Liquid Interfaces (Plenum, New York, 1989). 
${ }^{18}$ Y. M. Hifeda and G. W. Rayfield, J. Colloid Interface Sci. 104, 209 (1985).

${ }^{19}$ R. E. Heikkila, C. N. Kwong, and D. G. Cornwell, J. Lipid Res. 11, 190 (1970).

${ }^{20}$ T. Kato, Y. Hirobe, and M. Kato, Langmuir 7, 2208 (1991).

${ }^{21}$ M. L. Schlossman, D. K. Schwartz, P. S. Pershan, E. H. Kawamoto, G. J. Kellogg, and S. Lee, Phys. Rev. Lett. 66, 1599 (1991).

${ }^{22}$ D. K. Schwartz, M. L. Schlossman, and P. S. Pershan, J. Chem. Phys. 96, 2356 (1991)

${ }^{23}$ Joseph T. Buontempo, Stuart A. Rice, S. Karaborni, and J. I. Slepman, Langmuir 9, 1604 (1993)

${ }^{24}$ B. Lin, J. B. Peng, J. B. Ketterson, P. Dutta, B. N. Thomas, J. Buontempo, and S. A. Rice, J. Chem. Phys. 90, 2393 (1989).

${ }^{25}$ W. J. Foster, M. C. Shih, P. S. Pershan, MRS Symposium Proceedings 375, 187 (1995).

${ }^{26}$ B. Berge, O. Konovalov, J. Lajzerowicz, A. Renault, J. P. Rieu, and M. Vallade, Phys. Rev. Lett. 73, 1652 (1994).

${ }^{27}$ D. K. Schwartz, Ph.D. Thesis, Harvard University, 1991.

${ }^{28}$ W. J. Foster, Ph.D. Thesis, Harvard University, 1995.

${ }^{29}$ M. L. Schlossman and P. S. Pershan, in Light Scattering by Liquid Surfaces and Complementary Techniques, edited by D. Langevin (Marcel Dekker, New York, 1990).

${ }^{30}$ A. Braslau, Ph.D. thesis, Harvard University, 1988.

${ }^{31} \mathrm{~J}$. Als-Nielsen and K. Kjaer, in Phase Transitions in Soft Condensed Matter, edited by T. Riste and D. Sherrington (Plenum Press, New York, 1989), p. 113.

${ }^{32} \mathrm{P}$. Dutta, in Phase Transitions in Surface Films, edited by H. Taub, G. Torzo, H. Lauter, and S. Fain (Plenum Publishing Co., New York, 1991).

${ }^{33}$ X. Qiu, J. Ruiz-Garcia, and C. M. Knobler, Mat. Res. Soc. Symp. Proc. 237, 263 (1992).
${ }^{34}$ M. C. Shih, T. M. Bohanon, J. M. Mikrut, P. Zschack and P. Dutta, Phys. Rev. A 45, 5734 (1992).

${ }^{35} \mathrm{M}$. Bommarito et al. (unpublished).

${ }^{36}$ S. W. Barton, B. N. Thomas, E. B. Flom, S. A. Rice, B. Lin, J. B. Peng, J. B. Ketterson, and P. Dutta, J. Chem. Phys. 89, 2257 (1988).

${ }^{37}$ D. M. Small, The Physical Chemistry of Lipids (Plenum, New York, 1986).

${ }^{38}$ A. I. Kitaigorodskii, Organic Chemical Crystallography (Consultants Bureau, NY, 1961).

${ }^{39}$ X. Z. Wu, E. B. Sirota, S. K. Sinha, B. M. Ocko, and M. Deutsch, Phys. Rev. Lett. 70, 958 (1993)

${ }^{40}$ X. Z. Wu, B. M. Ocko, E. B. Sirota, S. K. Sinha, M. Deutsch, B. H. Cao, and M. W. Kim, Science 261, 1018 (1993).

${ }^{41}$ J. C. Earnshaw and C. J. Hughes, Phys. Rev. A 46, R4494 (1992).

${ }^{42}$ X. Z. Wu, E. B. Sirota, S. K. Sinha, B. M. Ocko, and M. Deutsch, Phys. Rev. Lett. 70, 958 (1993).

${ }^{43}$ I. Jalal, J. Colloid Interface Sci. 68, 196 (1979).

${ }^{44}$ A. P. Young, Phys. Rev. B 19, 1855 (1979).

${ }^{45}$ B. I. Halperin and D. R. Nelson, Phys. Rev. Lett. 41, 121 (1978).

${ }^{46}$ D. R. Nelson and B. I. Halperin, Phys. Rev. B 19, 2457 (1979).

${ }^{47}$ L. D. Landau, Phys. Z. Sowjet. 11, 26 (1937).

${ }^{48}$ B. Fischer, M. W. Tsao, J. Ruiz-Garcia, T. M. Fischer, D. K. Schwartz, and C. M. Knobler, J. Phys. Chem. 98, 7430 (1994).

${ }^{49}$ V. M. Kaganer, I. R. Peterson, R. M. Kenn, M. C. Shih, M. Durbin, and P. Dutta, J. Chem. Phys. 102, 9412 (1995).

${ }^{50} \mathrm{M}$. Bommarito et al. (unpublished).

${ }^{51}$ P. Tippmann-Kjaer and H. Mohwald, Langmuir 7, 2303 (1991). 